\title{
1 Q-SNARE Syntaxin 7 confers actin-dependent rapidly 2 replenishing synaptic vesicles upon high activity
}

4 Yasunori Mori ${ }^{1, \#}$, Koichiro Takenaka ${ }^{1}$, Yugo Fukazawa ${ }^{2}$ and Shigeo Takamori ${ }^{1, \#}$ 5

61 Laboratory of Neural Membrane Biology, Graduate School of Brain Science, 7 Doshisha University, Kyoto, Japan

$8{ }^{2}$ Division of Brain Structure and Function, Faculty of Medical Science, University of 9 Fukui, Fukui, Japan

10 \# All correspondence should be addressed to Y.M. (yasumori@mail.doshisha.ac.jp) or 11 S.T. (stakamor@mail.doshisha.ac.jp) 


\section{Abstract}

Replenishment of readily releasable synaptic vesicles (SVs) with vesicles in the recycling pool is important for sustained transmitter release during repetitive stimulation. Kinetics of replenishment and available pool size define synaptic performance. However, whether all SVs in the recycling pool are recruited for release with equal probability is unknown. Here, using comprehensive optical imaging for various presynaptic endosomal SNARE proteins in cultured hippocampal neurons, we demonstrate that part of the recycling pool bearing the endosomal Q-SNARE Syntaxin 7 (Stx7) is preferentially mobilized for release during high-frequency repetitive stimulation. Recruitment of the SV pool marked with the Stx7-reporter requires high intra-terminal $\mathrm{Ca}^{2+}$ concentrations and actin polymerization. Furthermore, disruption of Stx7 function by overexpressing the N-terminal domain selectively abolished this pool. Thus, our data indicate that endosomal membrane fusion involving Stx7 is essential for adaptation of synapses to respond high-frequency repetitive stimulation.

Hundreds to tens of thousands of neurotransmitter-filled synaptic vesicles (SVs), which are morphologically homogeneous, are clustered at presynaptic terminals, potentially capable of releasing their contents upon the arrival of electrical stimuli (Sudhof, 2004). Yet, only a fraction of all SVs is usually available for stimulus-dependent release (Alabi \& Tsien, 2012; Rizzoli \& Betz, 2005) (but see, Ikeda \& Bekkers, 2009). During the last few decades, the three-pool model has emerged, in which neurotransmitterfilled synaptic vesicles are classified into three functionally distinct pools: the readily releasable pool (RRP), the recycling pool that replenishes the RRP during sustained activity, and the reserve pool (RP, also referred to as the resting pool) that rarely participates in recycling (Alabi \& Tsien, 2012; Rizzoli \& Betz, 2005). Despite intensive research, however, the molecular underpinnings of the three pools have remained elusive.

Exocytic fusion of SVs to the plasma membrane is mediated by formation of socalled SNAP-receptor (SNARE) complexes, consisting of vesicular SNARE synaptobrevin (Syb)/VAMP2 and two target SNAREs Syntaxin 1 (Stx1) and SNAP-25 (Jahn \& Scheller, 2006). Considering the extraordinary speed of SV exocytosis in 
47 response to action potentials (typically within several ms), RRP is believed to dock physically to the plasma membrane prior to fusion and the canonical neuronal SNAREs described above are responsible for their docked status (Alabi \& Tsien, 2012; Rizzoli \& Betz, 2005). Aside from the necessity of the dominant SNAREs for the final step of SV exocytosis, non-canonical SNAREs that mediate fusion reactions between intracellular organelles in non-neuronal cells are not only present, but also enriched in SVs purified from rat brains (Takamori et al, 2006)(Appendix Fig S1), albeit at lower expression levels than the canonical SNAREs (Wilhelm et al, 2014), making them likely candidate protein constituents in functionally distinct subsets of SVs, such as the recycling and reserve pools. Indeed, endosomal SNAREs are sorted to distinct vesicle pools, namely in RP or spontaneously releasing vesicles (VAMP7 or vti1a), both of which are somewhat refractory to evoked release (Hua et al, 2011; Ramirez et al, 2012). Similarly, several endosomal SNAREs including vti1a, Stx6, and Stx12/13, also engage in SV recycling and may supply RRP (Hoopmann et al, 2010), and VAMP4 is directed to asynchronous SV pools (Raingo et al, 2012). However, whether and/or to what extent these presynaptic endosomal SNAREs are present within the recycling pool and consequently confer unique properties upon it, have not been determined.

Aside from the three functionally distinct SV pools described above, accumulating evidence suggests that even within the same presynaptic terminals, distinct types of SVs coexist. For instance, vesicular glutamate transporters (VGLUTs) and vesicular monoamine transporter 2, which are responsible for uptake of glutamate and biogenic monoamines, respectively, into SVs, are present on different populations of SVs (Onoa et al, 2010; Silm et al, 2019). In addition to their distinct exocytosis kinetics at a fixed stimulation frequency, exocytotic kinetics of respective SVs during repetitive stimulation appeared to differ depending on stimulation frequencies (Silm et al, 2019), suggesting the existence of various populations of SV recycling pools with distinct release probabilities. Although SV biogenesis mediated by adaptor protein (AP) -3 is a key step in formation of functionally distinct SV pools (Silm et al, 2019), it remains unknown whether differences in their molecular compositions are due to minor SV proteins, such as presynaptic endosomal SNAREs.

To gain deeper insights into the contribution of endosomal SNAREs in SV recycling, we monitored behaviors of presynaptic endosomal SNAREs conjugated C-terminally with pHluorin (also referred to as SEP)(Miesenböck et al, 1998; Sankaranarayanan et al, 
80 2000) in a comprehensive manner in response to various stimulation protocols. We 81 found that, among endosomal SNAREs, Stx7 is exclusively sorted to subpopulation of 82 the SV recycling pool which responds preferentially to high-frequency repetitive 83 stimulation. Rapid recruitment of this pool is driven by high $\mathrm{Ca}^{2+}$ concentrations and 84 requires actin dynamics, resembling a previously reported SV population that rapidly 85 replenishes RRP after synaptic depression (Alabi \& Tsien, 2012). Notably, blockade of 86 Stx7 function by overexpressing the $\mathrm{N}$-terminal domain selectively abolishes this pool. 87 Thus, our results collectively indicate a hitherto unrecognized mechanism by which the 88 rapidly replenishing SV recycling pool that sustains synaptic transmission during high89 frequency stimulation is generated by endosomal fusion events involving Stx 7 during 90 SV recycling. 


\section{$91 \quad$ Results}

92 Endosomal SNARE-SEPs localize in distinct types of presynaptic vesicle 93 compartments

94 To explore recycling properties of SVs carrying presynaptic endosomal SNAREs under 95 various stimulation protocols, we utilized a pHluorin reporter (super-ecliptic pHluorin: 96 SEP) fused to the luminal C-terminal portion of individual SNARE proteins (Fig 1A). 97 These include five Syntaxin (Stx) family members (Stx6, 7, 8, 12/13, and 16), all of 98 which were highly enriched in pure SV fraction (Takamori et al, 2006) (Appendix Fig 99 S1). We also included two additional endosomal SNAREs, vtila and VAMP7, which 100 reportedly mediate spontaneous release rather than stimulus-dependent evoked release 101 (Hua et al, 2011; Ramirez et al, 2012). As a control for authentic SV residents, two widely used SEP constructs, SypHy (Synaptophysin fused with SEP) (Fig 1A) and

103 VGLUT1-SEP were used (Granseth et al, 2006; Voglmaier et al, 2006). The SEP reporters have been widely used to monitor $\mathrm{SV}$ recycling, owing to their $\mathrm{pH}$ dependence by which SEP fluorescence is quenched in an acidic lumen of SVs, and de-quenched upon exposure to an extracellular neutral $\mathrm{pH}$ by exocytosis (Figs 1B-D). When SypHy was lentivirally transduced into cultured hippocampal neurons (Egashira et al, 2015, 2016), it was sorted to presynaptic compartments, evidenced by immunostaining with anti-Syb2 antibody (Fig 1B). When neurons were electrically stimulated repetitively at 10 or $40 \mathrm{~Hz}$ (300 or 200 action potentials (APs)), SypHy fluorescence robustly

111 increased in response to the stimuli, and > 30-40\% of all SypHy molecules, estimated 112 by application of $50 \mathrm{mM} \mathrm{NH}_{4} \mathrm{Cl}$ at the end of the recordings, was engaged in exocytosis

113 by the end of stimulation (Figs 1D-F). On the other hand, although presynaptic 114 endosomal SNARE-SEPs were similarly co-localized with Syb2 (Fig 1e), the vast 115 majority of endosomal SNARE-SEPs showed relatively smaller responses compared to 116 SypHy (Figs 1E,F ; <25\%) and in some cases, responses upon 40-Hz stimulation were 117 significantly smaller than those elicited at $10 \mathrm{~Hz}$ (e.g. Stx8-SEP, Stx16-SEP) (Figs 118 1E,F). Strikingly though, Stx7-SEP exhibited unique behavior, i.e. stimulation at $10 \mathrm{~Hz}$ 119 failed to elicit a clear fluorescence increase, while $40-\mathrm{Hz}$ stimulation caused a robust 120 increase up to $40 \%$ (Figs 1E,F). Quantitative comparisons of all SEP-reporters in 121 response to $10-\mathrm{Hz}$ and $40-\mathrm{Hz}$ stimulation (with 200 APs) revealed that Stx7-SEP 122 exhibited $\sim 8$-fold fluorescence increase at $40 \mathrm{~Hz}$ compared to $10 \mathrm{~Hz}$ (Figs 1E,F). 123 Overall, these results indicate that different presynaptic endosomal SNARE-SEPs 
124 localize to distinct membrane compartments that are capable of recycling in an

125 activity-dependent manner, to different degrees, at presynaptic terminals.

127 Only Stx7-SEP exclusively localizes to Syb2-positive SVs

128 Given distinct recycling behaviors of the presynaptic endosomal SNARE-SEPs 129 compared to SypHy, we wondered to what extent these endosomal SNARE-SEPs are 130 sorted into functionally fusion competent SVs. To test this, SEP responses were 131 monitored after pretreatment with tetanus toxin (TeNT) that enzymatically cleaves 132 Syb2/VAMP2 (Schiavo et al, 1992), thereby terminating SV exocytosis (Schoch et al, 133 2001). The robust response of Stx7-SEP at $40 \mathrm{~Hz}$ was completely blocked by TeNT 134 treatment, as was observed for SypHy and VGLUT1-SEP, indicating that Stx7-SEP was sorted into Syb2/VAMP2-positive, functionally competent SVs (Fig 1G; Appendix Fig S2). By contrast, responses of other endosomal SNARE-SEPs were not completely abolished by TeNT treatment, but were only diminished to varying extents (Fig 1G, Appendix Fig S2). We also examined luminal pH of intracellular compartments bearing SEP-reporters by sequential application of an acidic solution $(\mathrm{pH} 5.5)$ and a $50 \mathrm{mM}$ $\mathrm{NH}_{4} \mathrm{Cl}$ solution (Mitchell \& Ryan, 2004), and subsequent electrical stimulation to ensure active synapses (Fig 1H, Appendix Fig S3). These analyses revealed that the vesicular $\mathrm{pH}$ of Stx7-laden vesicles $(6.09 \pm 0.05)$ was identical to those of SypHy (5.96 $\pm 0.04)$ and VGLUT1-SEP $(5.98 \pm 0.05)$, whereas those of other endosomal SNARESEP-laden vesicles were significantly higher (> 6.40) (Fig $1 \mathrm{H})$. Thus, these results collectively demonstrate that, among presynaptic endosomal SNAREs, Stx7 is peculiar in that it exclusively localizes to a subpopulation of genuine fusion-competent SVs of the recycling pool that respond preferentially to high-frequency stimulation.

Stx7 localizes to a small portion of SV clusters with low copy numbers at the presynaptic terminals

151 In order to gain further evidence for localization of Stx7 in a subpopulation of SVs, we 152 adopted several different approaches. First, we co-stained cultured hippocampal neurons 153 with antibodies against Stx7 and Syp, and observed them using confocal microscopy 154 (Fig 2A). Although Stx7 immunoreactivities were apparent in cell somas and dendrites 155 as well (Appendix Fig S4), they were also observed along axons, and partially overlapped with Syp signals (Fig 2A), indicating that intrinsic Stx7 is localized within a 
157 cluster of SVs at presynaptic terminals. To examine detailed localization of Stx7 within

158 the terminals, we next performed immunoelectron microscopy. Since our initial trials to

159 detect endogenous Stx7 with the same antibody did not produce reliable signals, we

160 expressed Stx7-SEP as performed for SEP imaging, and proceeded to immunostaining 161 using anti-se antibody. Whereas SypHy signals with the same detection method were 162 evenly spread all over SV clusters in the terminals, Stx7-SEP signals were relatively 163 sparse and restricted to an area within SV clusters (Fig 2B). Notably, in many instances, 164 Stx7-SEP signals were located distant from active zone structures, which is consistent 165 with a previous observation under STED microscopy (Wilhelm et al, 2014).

166 Stx7 content in the synaptosomal fraction isolated from rat brains was reported to 167 be 78.6 copies per synapse ${ }^{7}$. However, the synaptosomal fraction also contains postsynaptic compartments in which Stx7 might be present (Appendix Fig S4). To estimate a fractional contribution of presynaptic Stx7 in the synaptosomal fraction, we quantified the Stx 7 content in purified SVs where Stx7 was shown to be highly enriched by western blot analysis (Takamori et al, 2006). We estimated the copy number of Stx 7 molecules per SV as $0.14 \pm 0.03$ (Figs 2C, D and Appendix Fig S5). Assuming that an average synaptosome contains $\sim 380 \mathrm{SVs}$, total Stx7 molecules located on SVs is

174 calculated to be $\sim 53$, which roughly coincides with the number of Stx 7 molecules in the synaptosomal fraction (Wilhelm et al, 2014), suggesting that more Stx7 molecules are present in presynaptic terminals than in postsynaptic compartments.

\section{Stx7-SEP is sorted to SV subpopulation that requires HFS for exocytosis}

179 One of the most striking features revealed by our comprehensive SEP imaging analysis

180 (Figs 1E,F) was that Stx7-SEP responded preferentially to high-frequency stimulation 181 (HFS) at $40 \mathrm{~Hz}$, but rarely responded to $10-\mathrm{Hz}$ stimulation. To confirm this 182 phenomenon and to avoid any bias possibly due to heterogeneity intrinsic to each 183 bouton or culture preparation, we continuously monitored changes in SEP fluorescence 184 at individual boutons with various stimulation frequencies ranging from $5 \mathrm{~Hz}$ to $40 \mathrm{~Hz}$ 185 at 5 min intervals (Fig 3A). Unlike SypHy, which reliably exhibited a robust exocytotic 186 fluorescence increase irrespective of stimulation frequency (Fig 3A, top), Stx7-SEP 187 hardly responded during $5-\mathrm{Hz}$ or $10-\mathrm{Hz}$ stimulation, while it showed robust 188 fluorescence increases at $20 \mathrm{~Hz}$ and $40 \mathrm{~Hz}$ in the same boutons (Fig 3A, bottom). 
We then wondered if overexpression of Stx7-SEP simply attenuated SV exocytosis generally, for instance by inactivating $\mathrm{Ca}^{2+}$ channels, or if Stx7-SEP localized at non-synaptic areas where non-SV type secretory vesicles that responded only to HFS were present. To exclude these possibilities, we co-expressed Stx7-SEP and Syp-mOr, in which a $\mathrm{pH}$-sensitive orange fluorescent protein, mOrange2, was fused to the luminal region of synaptophysin, instead of SEP (Egashira et al, 2015, 2016), and monitored their fluorescence changes simultaneously in the same presynaptic boutons (Appendix Fig S6). When analyses were restricted in bouton-like structures where clear fluorescence increases of Syp-mOr were detected, Stx7-SEP rarely responded to $10-\mathrm{Hz}$ stimulation (Appendix Fig S6), but exhibited drastic increase in response to $40-\mathrm{Hz}$ stimulation (Appendix Fig S6).

We next wondered if more prolonged stimulation at low frequencies $(5 \mathrm{~Hz}$ and 10 $\mathrm{Hz}$ ) could induce exocytosis of Stx7-SEP-laden vesicles. Since endocytosis and subsequent re-acidification of SVs during prolonged stimulation might mask the SEP fluorescence increase due to SV exocytosis (Kim \& Ryan, 2009), we blocked reacidification of endocytosed vesicles with $2 \mu \mathrm{M}$ bafilomycin A1 (Baf), a potent inhibitor of vacuolar-type $\mathrm{H}^{+}$-ATPases (V-ATPases), to compare exocytic properties of SypHy and Stx7-SEP (Fig 3B). We adopted 600 action potentials (APs), because that was sufficient to induce exocytosis of the entire recycling pool in cultured hippocampal neurons (Kim \& Ryan, 2010). In case of SypHy, fluorescence signals plateaued at the same levels (about 40-50\%) irrespective of stimulation frequencies (5$40 \mathrm{~Hz}$ ) (Fig 3C). In stark contrast, Stx7-SEP exhibited similar exocytic properties as

211 SypHy at $20 \mathrm{~Hz}$ and $40 \mathrm{~Hz}$, whereas it showed much less and slower responses during 5

$212 \mathrm{~Hz}$ and $10 \mathrm{~Hz}$ (Fig 3C). Comparisons between SypHy and Stx7-SEP by replotting as a

213 function of stimulus numbers clearly showed that rise kinetics of Stx7-SEP were 214 identical to those of SypHy during 20- $\mathrm{Hz}$ and $40-\mathrm{Hz}$ stimulation, whereas those of 215 Stx7-SEP were much slower than SypHy at $5 \mathrm{~Hz}$ and $10 \mathrm{~Hz}$ (Fig 3D).

217 Stx7-vesicles represent a fraction of the recycling pool, recruitment of which 218 requires high $\mathrm{Ca}^{2+}$ and actin polymerization

219 The fact that Stx7-SEP responded preferentially to high repetitive stimulation $(>20 \mathrm{~Hz})$ 220 suggested that exocytosis of Stx7-SEP-bearing SVs would require a high concentration 221 of $\mathrm{Ca}^{2+}$. To test this hypothesis, we raised external $\mathrm{Ca}^{2+}$ from 2 to $8 \mathrm{mM}$, and examined 
222 whether even $10-\mathrm{Hz}$ stimulation would cause robust responses. The response of SypHy 223 was facilitated in the presence of $8 \mathrm{mM}$ (Fig 4A, left), as observed previously 224 (Chanaday \& Kavalali, 2018). Notably, Stx7-SEP also exhibited a robust exocytic 225 response to $10-\mathrm{Hz}$ stimulation when external $\mathrm{Ca}^{2+}$ was raised to $8 \mathrm{mM}$ (Fig 4A).

We next focused on the actin cytoskeleton, because actin polymerization has often 227 been implicated in exo-endocytosis of SVs in cultured hippocampal neurons (Wu et al, 228 2016) (but see Hua et al, 2011; Sankaranarayanan et al, 2003). In particular, $229 \mathrm{Ca}^{2+} /$ calmodulin-dependent fast SV replenishment after RRP depletion is also mediated 230 by an actin-dependent process in calyx of Held synapses (Sakaba \& Neher, 2001, 2003). 231 We therefore asked whether actin dynamics are involved in recruitment of Stx7-SEPbearing vesicles for release during HFS. In accordance with a previous report (Hua et al, 2011), actin depolymerization with latrunculin A (Lat-A: $5 \mu \mathrm{M})$ did not show remarkable effects on SypHy recycling during 10-Hz stimulation (Fig 4B). However, Lat-A significantly retarded the SypHy response upon $40-\mathrm{Hz}$ stimulation (Fig 4B, upper right), indicating that there exists a subset of SVs for which mobilization for release is actin-dependent during intense stimulation. This result is consistent with previous results reported using neurons in which actin isoforms were conditionally deleted (Wu et al, 2016). Notably, more pronounced reduction by Lat-A was observed for Stx7-SEP responses upon $40-\mathrm{Hz}$ stimulation (Fig 4B), indicating that Stx7-SEP

241 localizes to a subpopulation of recycling SVs for which recruitment for release is actin242 dependent. Lat-B, another actin polymerization inhibitor, also decreased the SypHy and 243 Stx7-SEP responses to similar extent at $40 \mathrm{~Hz}$, but not at $10 \mathrm{~Hz}$ (Appendix Fig S7). 244 Finally, when Stx7-SEP and SypHy were monitored during 10-Hz stimulation in the 245 presence of $8 \mathrm{mM} \mathrm{Ca}^{2+}$, Lat-A significantly reduced the responses of both (Fig 4C).

246 To further determine whether Lat-A treatment reduced the size of the total recycling 247 pool or whether it simply slowed SV recruitment for release, we extended stimulation 248 lengths in the presence of Baf to measure the size of the total recycling pool of SypHy249 laden vesicles (Fig 4D). As expected, kinetics measured as $\tau_{\text {exo }}$ and the total recycling 250 pool size measured as the plateau at the end of stimulation, of SypHy responses during 251 10-Hz stimulation were not affected by Lat-A (Figs 4D,E), indicating that 252 actin-dependent SV mobilization does not contribute significantly to SV mobilization at 253 10-Hz stimulation. In contrast, during 20-Hz stimulation, while plateaus of SypHy 
254 responses was not affected by Lat-A treatment, kinetics of SypHy responses were 255 significantly slowed by Lat-A (Figs 4D,E).

256 Taken together, these results indicate that a portion of the SV recycling pool is 257 recruited through a pathway requiring $\mathrm{Ca}^{2+}$-dependent actin polymerization, and that

258 Stx7-SEP preferentially localizes to that pool.

The $\mathbf{N}$-terminal domain of $\mathbf{S t x} 7$ is responsible for its characteristic behavior

261 Like other Syntaxin family members, Stx7 comprises an N-terminal domain (NTD), the

262 SNARE domain and a transmembrane domain (TMD) (Jahn \& Scheller, 2006) (Fig 5A).

263 To understand the molecular mechanism by which Stx7 is selectively sorted to a 264 subpopulation of the SV recycling pool, we constructed two deletion mutants either 265 lacking the NTD (Stx7- - NTD-SEP) or the SNARE motif (Stx7- $\Delta$ SNARE-SEP) (Fig 266 5A). When these mutants were expressed in cultured neurons, Stx7-ANTD-SEP was properly sorted to Syb2-positive bouton-like puncta (Fig 5B) in which it preferentially localized to the intracellular acidic compartments, with albeit higher luminal $\mathrm{pH}$ compared to genuine SVs (Figs 5C,D and Appendix Fig S8). However, unlike Stx7SEP, Stx7- 7 NTD-SEP responded to $10-\mathrm{Hz}$ stimulation as it did to $40-\mathrm{Hz}$ stimulation

271 (Fig 5E) and TeNT treatment did not completely abolish the responses (Fig 5F).

272 Furthermore, responses of Stx7- $\triangle$ NTD-SEP at $40 \mathrm{~Hz}$ were no longer sensitive to Lat-A

273 treatment (Fig 5G). Thus, the NTD is essential for proper sorting of Stx7 to the actin-

274 dependent SV subpool that is preferentially recruited during HFS. By contrast, Stx7-

$275 \triangle$ SNARE-SEP did not accumulate at Syb2-positive bouton-like puncta, and was

276 distributed evenly on cell surfaces (Figs 5B-D and Appendix Fig S8), suggesting that

277 the SNARE domain is indispensable for its presynaptic, as well as vesicular,

278 localization.

\section{Overexpression of Stx7-NTD selectively reduces SV recruitment during HFS}

281 Although results described above demonstrate that Stx7-SEP is directed to a

282 subpopulation of the SV recycling pool for which recruitment for release requires high

$283 \mathrm{Ca}^{2+}$ and actin polymerization, whether Stx7 is necessary for this subpopulation of SVs 284 remains unclear, especially given that only a small population of SVs carries Stx7 (Fig 285 2). To address this question, we first examined whether silencing of Stx 7 with a specific shRNA affects the SV recycling sub-pool. However, chronic knockdown of Stx7 
287 expression severely reduced SypHy responses at $10 \mathrm{~Hz}$ and $40 \mathrm{~Hz}$, indicating that Stx7

288 is necessary to establish SV pool per se during synapse development and maturation

289 processes (Appendix Fig S9). As an alternative approach to inactivate Stx7, we

290 explored specific dominant-negative effects by overexpressing the $\mathrm{N}$-terminal domain

291 of Stx7. The rationale for this is that the NTD of Stx7 interacts with its own SNARE

292 motif, thereby inhibiting SNARE complex formation with cognate SNAREs (Antonin et

293 al, 2002). In addition, the results above (Fig 5) clearly demonstrate that the NTD of

294 Stx7 is essential for its proper sorting to the actin-dependent SV recycling pool. To this

295 end, we placed a P2A self-cleaving peptide between the SypHy and Stx7-NTD

296 sequences (a.a. 13-149) to ensure co-expression of both proteins (Egashira et al, 2016),

297 and monitored SV recycling reported by SypHy fluorescence in transfected neurons

298 (Fig 6A). Expression of Stx7-NTD did not alter the total recycling pool size or the

299 kinetics of vesicle release elicited at $10 \mathrm{~Hz}$ (Figs 6B, C). In stark contrast, expression of

300 the Stx7-NTD significantly slowed the increase of SypHy fluorescence during $20-\mathrm{Hz}$

301 stimulation, while total recycling pool size was affected only slightly (Figs 6B, C).

302 Treatment with Lat-A in addition to Stx7-NTD overexpression did not further slow

303 release kinetics (Fig 6D), indicating direct involvement of Stx7 in the actin-dependent

304 SV recycling pool that rapidly and preferentially replenishes RRP upon HFS. 


\section{Discussion}

308 Departing from comprehensive analyses of optical reporters for multiple presynaptic endosomal SNAREs, we report that an endosomal Q-SNARE, called Stx7, specifically

310 targets a unique SV pool that can replenish RRP only during intense activity.

311 Recruitment of this SV pool enabled by Stx7 requires high $\mathrm{Ca}^{2+}$ concentrations and

312 actin polymerization, characteristics conceptually resembling a population of the

313 recycling SV pool that rapidly replenishes RRP in calyx of Held synapses (Wu et al,

314 2016; Sakaba \& Neher, 2003; Lee et al, 2012; Piriya Ananda Babu et al, 2020). Our

315 results collectively indicate that endosomal fusion supported by Stx7, presumably

316 together with its cognate SNAREs, is responsible for generating a functionally distinct

317 recycling pool of SVs.

318

319 Recycling vesicle pool heterogeneity conferred by endosomal SNAREs

320 Previous studies using SEP constructs have suggested that release properties of a 321 subpopulation of SVs are dependent upon endosomal SNAREs (Hua et al, 2011;

322 Ramirez et al, 2012; Hoopmann et al, 2010; Raingo et al, 2012). For instance, the Qc-

323 SNARE, vtila, and the R-SNARE, VAMP7, reside mainly on the reserve pool that is

324 reluctantly mobilized during activity. Instead, SVs bearing these SNAREs are prone to

325 fuse spontaneously in the absence of activity (Hua et al, 2011; Ramirez et al, 2012).

326 Likewise, other endosomal SNAREs, Stx6, Stx12/13, and VAMP4 are capable of

327 recycling, albeit to a lesser extent than authentic SV proteins, such as Syb2(Hoopmann

328 et al, 2010; Raingo et al, 2012). However, our comprehensive SEP imaging analyses to

329 monitor the effect of TeNT on stimulus-dependent fluorescence changes, as well as

330 vesicular pHs of vesicle compartments on which they reside reveals that presynaptic

331 endosomal SNAREs can be categorized into two distinct classes; i.e., Stx7 and other

332 endosomal SNAREs. First, Stx7-SEP resides on a fraction of the recycling vesicle pool

333 that is preferentially mobilized during HFS (e.g. $>20 \mathrm{~Hz}$ ). Mobilization of this pool

334 depends on an increase in cytosolic $\left[\mathrm{Ca}^{2+}\right]$ exceeding a certain threshold, and also

335 requires actin dynamics (Fig. 4). Since Stx7-laden vesicles share some characteristic

336 features of genuine SVs, e.g., the same vesicular pH as SypHy- or VGLUT1-carrying

337 vesicles, and complete silencing of activity-dependent exocytosis by TeNT treatment,

338 we conclude that Stx7 resides on a subpopulation of the recycling SV pool. 
In great contrast to Stx7, other presynaptic endosomal SNAREs exhibit different characteristics and behaviors compared to genuine SV residents. While they responded to wide range of stimulation, their exocytotic responses were only partially attenuated

342 by TeNT treatment, indicating that these endosomal SNAREs are present, albeit to 343 various extents, on Syb2/VGLUT1-negative vesicle compartments. This is further 344 supported by the fact that the average vesicular $\mathrm{pH}$ of vesicles containing these 345 endosomal SNAREs appeared to be significantly higher ( 6.4) than the $\mathrm{pH}$ of authentic 346 SV residents. Thus, although it was proposed that Stx6, Stx12/13, and vti1a are 347 involved in replenishment of RRPs (Hoopmann et al, 2010), they are only minimally 348 present in the RRP and recycling SV pool. Specifically, they are present in non-SV compartments, which nevertheless recycle in an activity-dependent manner. Additionally, although a previous study proposed that vti1a selectively resides on Syb2free vesicles, which are preferentially utilized for spontaneous release rather than stimulus-dependent evoked release(Ramirez et al, 2012), our results reveal that the vast majority of presynaptic endosomal SNAREs are present on Syb2-free vesicles. The identity and function of such non-SV recycling vesicles at presynaptic terminals conferred by these endosomal SNAREs are enigmatic, but they may utilize noncanonical neuronal SNARE proteins and $\mathrm{C} \mathrm{Ca}^{2+}$ sensor for exocytotic fusion with the plasma membrane, such as SNAP-29, or -47 , Stx3 or 4, and Synaptotagmin-7 (Ibata et al, 2019; Jurado et al, 2013; Liu et al, 2014). Notably, recent studies have proposed the existence of non-SV-type secretory organelles at synaptic sites or in close proximity to presynaptic active zones, which secrete neuropeptides from lysosomes or dense-core vesicles (Ibata et al, 2019; Shimojo et al, 2015; Emperador-Melero et al, 2018). Unraveling SNAREs or $\mathrm{Ca}^{2+}$ sensors involved in these non-SV vesicles will enable future research to understand the physiological significance of these non-SV secretory organelles for synaptic performance and signaling.

\section{Actin and SV mobilization}

367 The role of the actin cytoskeleton in regulating synaptic vesicle mobilization has long 368 been controversial. While Lat-A, an actin polymerization inhibitor, had little impact on 369 SV mobility or recycling in hippocampal neurons (Hua et al, 2011; Sankaranarayanan et $370 a l, 2003)$, similar treatment resulted in a deceleration of SV replenishment of the RRP in 371 calyx of Held synapses (Sakaba \& Neher, 2003; Lee et al, 2012; Piriya Ananda Babu et 
$372 a l, 2020$ ), indicating the presence of actin-dependent rapidly replenishing SVs. Here,

373 using SypHy imaging, we provide evidence compatible for both views regarding the

374 role of actin dynamics in SV exocytosis and recruitment. Specifically, SVs are recruited

375 for release in an actin-independent manner during mild stimulation, while exocytotic

376 release of a significant fraction of SVs is accelerated by an actin-dependent process

377 during high frequency stimulation (Figs 4B,D, and E). The former is consistent with

378 previous observations (Hua et al, 2011; Sankaranarayanan et al, 2003) and the latter is

379 compatible with reduced SV replenishment into the RRP proposed in calyx of Held 380 synapses, reportedly mediated by $\mathrm{Ca}^{2+} /$ Calmodulin-dependent processes (Sakaba \&

381 Neher, 2001). Interestingly, SVs carrying Stx7-SEP are preferentially utilized for release during intense activity and are more dependent on actin dynamics than the remainder of the recycling pool revealed by SypHy. Furthermore, recruitment of Stx7SEP-laden SVs requires high intra-terminal $\mathrm{Ca}^{2+}$. Thus, our results collectively indicate that SVs characterized by Stx7 may represent a fraction of recycling SV pool that is rapidly recruited to replenish the RRP in an actin-dependent manner during high activity.

\section{Possible roles of Stx7 to confer the actin-dependent rapidly replenishing SVs}

390 How does Stx7 contribute to rapidly replenishing SVs? In non-neuronal cells, Stx7 is 391 required for homotypic/heteromeric fusion of late-endosomes and lysosomes (Mullock et al, 2000; Antonin et al, 2000; Ward et al, 2000) or lysosomes and autophagosomes (Matsui et al, 2018), and this process contributes to protein sorting and organelle maturation (He et al, 2016). In accordance with the role of Stx7 in non-neuronal cells, we propose that Stx7 may contribute to formation of the actin-dependent SV subpool by mediating homotypic as well as heterotypic fusion with existing endosomal structures. Recent evidence suggests that generation of clathrin-independent large endosome-like vacuoles or bulk endocytosis is the predominant form of endocytosis, especially after repetitive stimulation (Kononenko et al, 2014; Nicholson-Fish et al, 2015). Although current models predict clathrin-mediated SV reformation directly from

401 these endosomal membranes, it is also evident that homotypic or heterotypic fusion of 402 endosomes mediated by endosomal SNAREs can occur before SV reformation, even 403 with multiple rounds during high activity (Holroyd et al, 1999; Rizzoli et al, 2006).

404 Although a molecular link between Stx7 and actin is enigmatic, Stx7 may help generate 
405 the rapidly replenishing SV pool as a result of endosomal fusion and molecular sorting.

406 This model seems to work if Stx7, but not other cognate SNAREs, are destined to SVs,

407 and if exogenously expressed Stx7-SEP follows the trafficking route of endogenous

408 Stx7.

409 Some other scenarios may explain the contribution of Stx7 to the observed results.

410 First, it is possible that Stx7 directly or indirectly associates with actin filaments and

411 rapid recruitment of Stx7-vesicles is only possible when the $\mathrm{Ca}^{2+} /$ calmodulin cascade is

412 activated. Alternatively, Stx7 may contribute to compound membrane fusion with its

413 cognate SNAREs, involving fusion of multiple vesicles prior to a final fusion(He et al,

414 2009). However, considering the limited number of copies of Stx7 in a subpopulation of

415 SVs (only $14 \%$ of SVs can harbor a single copy of Stx7) and the relatively high impact

416 of Stx7-NTD overexpression on the SypHy response, as well as the highly specific

417 behavior of Stx7 among presynaptic endosomal SNAREs, these are not likely

418 possibilities. Further studies will be necessary to determine how Stx7, presumably with

419 its cognate SNAREs, functionally differentiates this SV pool, thereby affecting activity-

420 dependent synaptic performance. 


\section{Methods}

422

\section{Molecular Biology}

424 SypHy, Syp-mOr, VGLUT1-SEP, and endosomal SNARE-SEPs were expressed in a

425 neuron-specific manner using lentivirus-based vectors, in combination with the Tet426 Off system (Egashira et al, 2015, 2016). Two vectors were used, a 'regulator' vector 427 expressing an advanced tetracycline transactivator (tTAad) under the control of the 428 human synapsin 1 promoter (pLenti6PW-STB), and a "response" vector that expressed 429 SypHy, Syp-mOr, VGLUT1-SEP or endosomal SNARE-SEPs under the control of a 430 modified tetracycline-response element (TRE) composite promoter (pLenti6PW-TRE).

431 To construct endosomal SNARE-SEPs and deletion mutants, mouse coding sequences 432 for Stx6 (accession no. NM_021433.3), Stx7 (accession no. NM_016797.4), Stx8 433 (accession no. NM_018768.2), Stx12/13 (accession no. NM_018768.2), Stx16C 434 (accession no. NM_001102424.1), vtila (accession no. NM_001293685.1), VAMP4 435 (accession no. NM_016796.3), VAMP7 (accession no. NM_001302138.1), Stx7- $\Delta \mathrm{N}$ 436 (a.a.168-261) and Stx7- $\operatorname{SNARE~(a.a.1-167,~228-261)~without~the~stop~codons~were~}$ 437 amplified by PCR using mouse adult brain complementary DNA as a template, and 438 fused with super-ecliptic pHluorin (SEP). Thereafter, they were cloned into a SmaI site 439 located downstream of the TRE sequence in pLenti6PW-TRE using an In-Fusion 440 Cloning Kit (Clontech)(Egashira et al, 2015, 2016). VGLUT1-SEP was constructed as 441 described previously(Voglmaier et al, 2006) and cloned into the SmaI site of 442 pLenti6PW-TRE. SypHy and Syp-mOr plasmids were generated as described 443 previously (Egashira et al, 2015, 2016). To co-express SypHy and the N-terminal 444 domain of Stx7 (Stx7-NTD; a.a.13-137), a sequence encoding a self-cleaving P2A 445 peptide was placed between SypHy and Stx7-NTD(Egashira et al, 2016). To generate 446 recombinant proteins as standards for quantification analysis, Stx7-NTD (a.a. 1-149) 447 and Syb2-_TMD (a.a. 1-94) (accession no. NM_009497.3) were amplified by PCR, 448 and cloned into the BamHI site and SalI site of pGEX6P-1. Lentiviral vectors carrying 449 mCherry and either scrambled shRNA (CCTAAGGTTAAGTCGCCCTCG), mouse 450 Stx7 shRNA-\#1 (CGATATGATTGACAGCATAGA), or mouse Stx7 shRNA-\#2; 451 (GAAGCTGATATTATGGACATT) were obtained from VectorBuilder biotechnology 452 Co. Ltd. 


\section{Lentiviral vector production}

455 Lentiviral vectors to express SEP constructs as well as shRNAs for Stx7 in cultured 456 neurons were produced in human embryonic kidney (HEK) 293T cells, as described 457 previously (Egashira et al, 2015, 2016). Briefly, HEK293T cells cultured in a 75- $\mathrm{cm}^{2}$ 458 flask (Falcon) at $\sim 50 \%$ confluency were transfected with $17 \mu \mathrm{g}$ lentiviral backbone 459 plasmids (either pLenti6PW-STB or pLenti6PW-SEP reporters) and helper plasmids 460 (10 $\mu \mathrm{g}$ pGAG-kGP1, $5 \mu \mathrm{g}$ pCAG-RTR2, and $5 \mu \mathrm{g}$ pCAG-VSVG) using a calcium 461 phosphate transfection method (Chen \& Okayama, 1987). 16-24 h after transfection, 462 culture medium was replaced with $7 \mathrm{~mL}$ of fresh Neurobasal medium supplemented 463 with $2 \%$ B27 and $0.5 \mathrm{mM}$ glutamine. Supernatants were collected $48 \mathrm{~h}$ later, and 464 filtered through a $22-\mu \mathrm{m}$ filter unit to remove cell debris. Aliquots were flash-frozen in 465 liquid nitrogen and stored at $-80^{\circ} \mathrm{C}$ until use.

466

467 Neuronal culture and viral expression

468 Primary hippocampal cultures were prepared from embryonic day 16 ICR mice, as 469 described previously (Egashira et al, 2015, 2016), with slight modifications. Briefly, 470 hippocampi were dissected, and incubated with papain (90 units/mL, Worthington) for $47120 \mathrm{~min}$ at $37^{\circ} \mathrm{C}$. After digestion, hippocampal cells were plated onto poly-D-lysine472 coated coverslips framed either in 24 well plates, 12 well plates (Falcon), or a Nunc 4 473 well dish (Thermo fisher) at a cell density of 20,000-30,000 cells $/ \mathrm{cm}^{2}$, and kept in a 5\% $474 \mathrm{CO}_{2}$ humidified incubator. At 2-4 days in vitro (DIV), $40 \mu \mathrm{M}$ FUDR (Sigma) and 100 $475 \mu \mathrm{M}$ uridine (Sigma) were added to inhibit the growth of glial cells. One-fifth of the 476 culture medium was routinely replaced with fresh medium every 2-4 days until 477 recordings. Cultures were transduced with two lentiviral vectors, the regulator vector 478 and response vectors that encode SEP reporters or shRNAs for Stx 7 at between 2 to 7 479 DIV, and were subjected to experiments at 12-14 DIV.

\section{$481 \quad$ Live imaging}

482 Fluorescence imaging was carried out at room temperature $\left(\sim 27{ }^{\circ} \mathrm{C}\right)$ on an inverted 483 microscope (Olympus) equipped with a 60× (1.35 NA) oil immersion objective and 75484 W Xenon lamp. Cells cultured on a glass coverslip were placed in a custom-made 485 imaging chamber on a movable stage and continuously perfused with a standard extracellular solution containing (in mM): $140 \mathrm{NaCl}, 2.4 \mathrm{KCl}, 10$ HEPES, 10 glucose, 2 
$487 \mathrm{CaCl}_{2}, 1 \mathrm{MgCl}_{2}, 0.02 \mathrm{CNQX}$ and $0.025 \mathrm{D}-\mathrm{APV}(\mathrm{pH} 7.4)$. Field stimulation at various

488 frequencies from $5 \mathrm{~Hz}$ to $40 \mathrm{~Hz}$ (indicated in each figure) was delivered via bipolar 489 platinum electrodes with $1-\mathrm{ms}$ constant voltage pulses $(50 \mathrm{~V})$. At the end of recordings, 490 a extracellular solution containing (in $\mathrm{mM}$ ): $50 \mathrm{NH}_{4} \mathrm{Cl}, 90 \mathrm{NaCl}, 2.4 \mathrm{KCl}, 10 \mathrm{HEPES}$, 49110 glucose, $2 \mathrm{CaCl}_{2}, 1 \mathrm{MgCl}_{2}$ (pH 7.4) was applied directly onto the area of interest 492 with a combination of a fast flow exchange microperfusion device and a bulb controller, 493 both of which were controlled by Clampex 10.2. To measure kinetics of exocytosis and 494 total recycling pool size, neurons were treated with a standard extracellular solution 495 containing $2 \mu \mathrm{M}$ bafilomycin $\mathrm{A} 1$ (Baf) (BioViotica) for $1 \mathrm{~min}$, and placed in the 496 imaging chamber. Baf-containing solution was continuously applied to neurons 497 throughout the recordings. For HFS, we adopted a stimulation frequency of $20 \mathrm{~Hz}$ 498 instead of $40 \mathrm{~Hz}$, since prolonged stimulation at $40 \mathrm{~Hz}$ often causes unavoidable air bubbles around stimulation electrodes, which hindered reliable imaging. For TeNT experiments, neurons were incubated with $10 \mathrm{nM}$ recombinant TeNT-light chain 501 (Sigma-Aldrich) for $16 \mathrm{~h}$ in a $\mathrm{CO}_{2}$ incubator. For Lat-A and Lat-B experiments, neurons were treated with either $5 \mu \mathrm{M}$ Lat-A (Wako) or $10 \mu \mathrm{M}$ Lat-B (Cayman Chemical) for $1 \mathrm{~min}$ at $\mathrm{RT}$, and subjected to imaging experiments. Lat-A or -B containing buffer was continuously supplied to neurons throughout the recordings.

To estimate luminal $\mathrm{pH}$ and the surface fraction of SEP probes, a low $\mathrm{pH}$ solution containing (in mM): $140 \mathrm{NaCl}, 2.4 \mathrm{KCl}, 10 \mathrm{MES}, 4 \mathrm{MgCl}_{2}$ (pH 5.5) and $50 \mathrm{mM} \mathrm{NH}_{4} \mathrm{Cl}$ solution described above were successively applied to cultured neurons as described previously(Egashira et al, 2015, 2016). To restrict analyses at active synaptic boutons, electrical stimulation at $40 \mathrm{~Hz}$ was applied for $10 \mathrm{~s}$ at the end of recordings.

510 Fluorescence images (1024 × 1024 pixels $)$ were acquired with ORCA-Flash 4.0

511 sCMOS camera (Hamamatsu Photonics) in time-lapse mode either at $1 \mathrm{~Hz}$ (for imaging 512 in response to stimulation) or $5.7 \mathrm{~Hz}$ (for estimating vesicular $\mathrm{pH}$ and surface fraction) 513 under control of MetaMorph software (Molecular Devices). Fluorescence of SypHy, 514 VGLUT1-SEP, or endosomal SNARE-SEPs was imaged with 470/22 nm excitation 515 and 514/30 nm emission filters, and Syp-mOr was imaged with 546/556 nm excitation 516 and 575/625 nm emission filters.

\section{Image data analysis}


519 Acquired fluorescence images were analyzed using MetaMorph software. Active

520 synapses were identified manually by changes in live measurements of fluorescence.

521 Circular region of interests (ROIs; $2.26 \mu \mathrm{m}$ diameter and $4 \mu \mathrm{m}^{2}$ area) were positioned

522 manually at the center of the highlighted fluorescence spots. Non-responsive puncta,

523 especially for presynaptic endosomal SNARE-SEPs, which became apparent only upon

$524 \mathrm{NH}_{4} \mathrm{Cl}$ application, were excluded from analysis, since they may represent endosomal

525 SNARE-SEPs expressed outside of presynaptic terminals. To extract fluorescence

526 changes associated with stimulation at each bouton, an average of five consecutive

527 fluorescence values was take as $F_{0}$, and this value was subtracted from the subsequent

528 fluorescence values $(\Delta \mathrm{F})$. To estimate relative fluorescence over total SEP molecules,

529 the peak value during $\mathrm{NH}_{4} \mathrm{Cl}$ application was taken as $\Delta \mathrm{F}_{\mathrm{NH}_{4} \mathrm{Cl}}\left(\mathrm{F}_{\mathrm{NH}_{4} \mathrm{Cl}}-\mathrm{F}_{0}\right)$ and used to

530 normalize the data $\left(\Delta \mathrm{F} / \Delta \mathrm{F}_{\mathrm{NH} 4 \mathrm{Cl}}\right)$. For each constructs, fluorescence changes of at least

53150 boutons were analyzed and expressed as mean \pm s.e.m. To calculate rise kinetics

532 of the fluorescence increase $\left(\tau_{\text {exo }}\right)$, an average trace from one image (containing $5-10$

533 active boutons) was collected, and taken as $n=1$. For each averaged trace, $\tau_{\text {exo }}$ was

534 calculated by fitting the trace with a single exponential function using a Solver function

535 from Excel software. Averages of time constants calculated from at least 10 images

536 were statistically evaluated.

537 Acquired images for vesicular $\mathrm{pH}$ and surface fraction measurements were also 538 processed as described above. Vesicular $\mathrm{pH}$ and surface fraction were calculated as 539 described previously (Egashira et al, 2015, 2016), except that fluorescence during $540 \quad \mathrm{NH}_{4} \mathrm{Cl}$ application was approximated as fluorescence at $\mathrm{pH}$ 7.4. Values of $\mathrm{p} K_{\mathrm{a}}(7.1)$ and

541 a Hill co-efficient (1.0) for SEP reported previously (Mitchell \& Ryan, 2004;

542 Sankaranarayanan et al, 2000) were used for calculation.

543

544 Immunocytochemistry

545 Cultured neurons at DIV12-14 were fixed with $4 \%$ (wt/vol) paraformaldehyde in 546 phosphate buffer (Wako) for $10 \mathrm{~min}$ at RT. After washing with PBS, cells were 547 permeabilized with PBS containing $0.2 \%$ Triton $\mathrm{X}-100$ for $20 \mathrm{~min}$ at RT, and incubated 548 with PBS containing 10\% (vol/vol) FBS for $30 \mathrm{~min}$ at RT. Cells were incubated with 549 rabbit polyclonal anti-GFP antiserum and mouse monoclonal anti-Syb2 antibody 550 (Cl69.1) (kind gifs from Reinhard Jahn) (Figs 1B,E, and 5B), or with rabbit polyclonal 551 anti-Stx7 antibody (Bethyl Laboratories; A304-512A) and mouse monoclonal anti- 
552 Synaptophysin antibody (Cl7.2)(Fig 2A), or chick polyclonal anti-MAP2 antibody 553 (1:1,000; Abcam; ab5392) and anti-Stx7 antibody (Appendix Fig S4), for $1 \mathrm{~h}$ at RT.

554 Cells were rinsed $3 \times$ with PBS, and further incubated with Alexa-488-conjugated anti555 rabbit $\operatorname{IgG}$ (1:1,500; Invitrogen), Alexa-555-conjugated anti-mouse $\operatorname{IgG}(1: 1,500$; 556 Invitrogen), or Alexa-633-conjugated anti-chick IgG (1:1,500; Invitrogen) for 30 min 557 at RT. Fluorescence images were acquired with an inverted microscope (Olympus) with 558 an ORCA-Flash 4.0 sCMOS camera (Hamamatsu Photonics) irradiated by 75-W 559 Xenon lamp (Alexa-488, 470/22 $\mathrm{nm}$ excitation and 514/30 nm emission filters; Alexa$560555,556-$ to $570-\mathrm{nm}$ excitation and 600- to 650-nm emission filter; Alexa-633; 624561 to $664-\mathrm{nm}$ excitation and $692-$ to $732-\mathrm{nm}$ emission filter) (Figs 1B,E, 5B, and 562 Appendix Fig S4). Fluorescence images (Fig 2A) were obtained with a laser scanning 563 confocal microscope equipped with a $100 \times(1.35 \mathrm{NA})$ oil immersion objective (LSM710; Zeiss), and images were collected with ZEN software.

565

566

\section{Immunoelectron microscopy}

567 Hippocampal neurons grown on a coverslip were transduced with lentiviruses carrying either SypHy or Stx7-SEP and were fixed for $2 \mathrm{~h}$ with $4 \%$ paraformaldehyde and $0.05 \%$ glutaraldehyde in phosphate-buffered saline (PBS; pH7.4) at 15 DIV. These neurons were processed for subcellular localization with an anti-GFP antiserum by employing pre-embedding immunoelectron microscopy. Briefly, plasma membranes of

572 fixed neurons were permeabilized with $0.1 \%$ Triton $\mathrm{X}-100$ in PBS for $15 \mathrm{~min}$ followed 573 by blocking with $10 \%$ fetal bovine serum in PBS for $30 \mathrm{~min}$ and then incubated with 574 rabbit anti-GFP antiserum (1:1,000 dilution in 10\% PBS) for $24 \mathrm{~h}$ at $4^{\circ} \mathrm{C}$. After several 575 washes with PBS, they were incubated with $1.4 \mathrm{~nm}$ immunogold-conjugated anti-rabbit $576 \operatorname{IgG}$ (Nanoprobe, 1:100 dilution in 2\% normal goat serum in PBS) overnight at $4^{\circ} \mathrm{C}$ and 577 then subjected to post-fixation with $1 \%$ glutaraldehyde in PBS for $10 \mathrm{~min}$. Immunogold 578 particles were enhanced for 7 min using the HQ silver enhancement kit (Nanoprobe) according to manufacturer instructions. After post-fixation with $1 \%$ osmium tetroxide in PB for 40 min on ice, these samples were stained with $1 \%$ uranyl acetate solution for

$58135 \mathrm{~min}$, dehydrated with an ethanol series and propylene oxide for $10 \mathrm{~min}$ each and then 582 embedded in Durcupan resin. After polymerization of the resin at $60{ }^{\circ} \mathrm{C}$ for $48 \mathrm{~h}$, 583 coverslips were removed and serial ultrathin sections $(40 \mathrm{~nm})$ were prepared. These 584 sections were observed under a transmission electron microscope at $100 \mathrm{kV}(\mathrm{H}-7650$, 
585 Hitachi Co., Japan) and digital images were taken with a Quemessa CCD camera

586 (Olympus-SIS, Germany).

587

\section{Protein purification}

589 Expression of GST-tagged Stx7-NTD or Syb2590 BL21 cells by adding $1 \mathrm{mM}$ isopropyl- $\beta-\mathrm{D}-$-thiogalactopyranoside (IPTG) at $37^{\circ} \mathrm{C}$ for $5914 \mathrm{~h}$ for GST-Stx7-NTD, or $0.1 \mathrm{mM}$ IPTG at $25^{\circ} \mathrm{C}$, overnight for GST-Syb2- $\Delta$ TMD.

592 Recombinant proteins were purified with glutathione-Sepharose 4 Fast Flow 593 (Amersham Biosciences) resin according to the manufacturer instructions. Protein 594 concentrations were determined by BCA Assay (Pierce).

595

596 Immunoblotting

597 Protein samples were separated on SDS-polyacrylamide mini-gels and blotted onto 598 PVDF membranes (Millipore). As primary antibodies, rabbit polyclonal anti-Stx7 599 (Bethyl Laboratories), mouse monoclonal anti-Syb2 (C169.1), and mouse monoclonal 600 anti-Tuj1 (Covance) were used. Blots were further incubated with secondary antibodies 601 (anti-mouse IgG or anti-rabbit IgG, respectively) coupled to horseradish-peroxidase 602 and developed using a Western Lightning Plus-ECL kit (PerkinElmer). Signals were 603 detected using a Molecular Imager ChemiDoc (BioRad).

604 To estimate the copy number of Stx7 per SV, CPG-purified SV fraction (a gift from 605 Reinhard Jahn) with known protein concentration and vesicle number, was subjected to 606 western blot together with various amounts of standard proteins for Stx7 and Syb2. 607 Band intensities in acquired images were quantified using Quantity One software 608 (BioRad).

609

610 Animals

611 Pregnant ICR mice were purchased from SLC, Japan. All mice were given food and 612 water ad libitum. Animals were kept in a local animal facility with a 12-h light and 12$613 \mathrm{~h}$ dark cycle. Ambient temperature was maintained around $21^{\circ} \mathrm{C}$ with a relative 614 humidity of 50\%. All animal experiments were approved by the Institutional Animal 615 Care and Use Committee of Doshisha University.

616

\section{Statistics}


618 All data are shown as the mean \pm standard error of the mean (SEM). Unpaired $t$-tests

619 were applied to compare means of two experimental groups. All statistical tests were

620 two-tailed, and the level of statistical significance is indicated by asterisks: ${ }^{*} p<0.05$,

$621 * * p<0.01, * * * p<0.001$. n.s.; not significant.

622

\section{Data availability}

624 All relevant data that support the findings of this study are available from the

625 corresponding authors upon request. The detailed data for Fig. 1E-H, 3D, E, 4, 5C-G, 626 and 6B-D are included in Source data file.

627

628 
Figure 1. Comprehensive monitoring of presynaptic endosomal SNARE-SEPs reveals their characteristic recycling behavior.

633 a, Cartoon representation of SypHy (Synaptophysin-SEP) and an endosomal-SNARE-

634 SEP. b-d, Strategies for comprehensive characterization of presynaptic SNARE-SEPs.

635 After SEP constructs were lentivirally transduced in cultured hippocampal neurons, 636 distributions of SEP-fused proteins and their responses to repetitive electrical 637 stimulation were analyzed by immunocytochemistry (b) and live fluorescence imaging 638 (c, d). Representative results obtained for SypHy are shown. The SEP was visualized 639 with anti-GFP antibody, whereas locations of presynaptic boutons were identified with 640 anti-synaptobrevin 2 (Syb2) -antibody. To estimate fractional responses of each SEP 641 construct, $50 \mathrm{mM} \mathrm{NH}_{4} \mathrm{Cl}(\mathrm{pH} 7.4)$ was applied at the end of recordings, which revealed 642 total expression of SEP-fused proteins at individual boutons. Scale bars indicate $5 \mu \mathrm{m}$ 643 in (b) and $2 \mu \mathrm{m}$ in (c). e, Synaptic localization and stimulus-dependent recycling of 644 endosomal SNARE-SEPs upon $10-\mathrm{Hz}$ or $40-\mathrm{Hz}$ stimulation. Left images are 645 representative images of each SEP-fused protein. Scale bar indicates $5 \mu \mathrm{m}$. Right traces 646 show average fluorescence of individual SEP-fused proteins upon $10-\mathrm{Hz}$ (300 APs; 647 black) and 40-Hz (200 APs; red) stimulation. Fluorescence was normalized to those 648 during $\mathrm{NH}_{4} \mathrm{Cl}$ application. Data are averages of $>50-200$ boutons. $\mathrm{f}$, Peak fluorescence 649 of SEP probes at 200APs of $10-\mathrm{Hz}$ (black) and $40-\mathrm{Hz}$ stimulation (red). g, Effect of 650 TeNT pretreatment on recycling of SEP probes in comparison to SypHy at $40-\mathrm{Hz}$ 651 stimulation (200 APs) (see also Appendix Fig S2). Data were obtained from >35-100 652 boutons. For bar graph comparisons, fluorescence peaks without TeNT preatment were 653 taken as $100 \%$. n.s $p>0.05$, ** $p<0.01$, *** $p<0.001$ compared to SypHy after TeNT 654 treatment (Student's $t$-test). h, Luminal pHs of vesicle compartments carrying SEP 655 probes calculated from experiments in Appendix Fig S3. *** $p<0.001$ compared to 656 that of SypHy (Student's $t$-test).

658 Figure 2. Stx7 localizes to a subpopulation of SVs at presynaptic terminals.

659 a, Double immunostaining by Stx7 and Synaptophysin (Syp) antibodies in cultured 660 hippocampal neurons at 14 DIV. An upper panel shows representative axonal 661 localization of Stx7 (green) and Syp (red). Magnified images of numbered areas (1, 2, 
662 and 3) are shown individually below. Merged images show that Stx7 signals occupied a

663 portion of Syp-positive puncta. Note that the specificity of the Stx7 antibody was

664 confirmed in independent experiments where Stx7 expression level was reduced by

665 specific shRNA (Appendix Fig. S9B). Scale bars indicate $2 \mu \mathrm{m}$. b, Electron

666 micrographs of SypHy and Stx7-SEP at presynaptic terminals. Representative electron

667 micrographs of a synaptic vesicle cluster labeled for SypHy (top) or Stx7-SEP (bottom)

668 at presynaptic terminal are shown. Immunogold labeling was intensified by silver

669 enhancement. Arrowheads indicate both ends of the active zone. Scale bars indicate 100

$670 \mathrm{~nm}$. c, A representative quantification of Stx7 in native SVs purified from rat brains.

671 Various amounts of recombinant GST-Stx7-N-terminal domain (GST-Stx7-N) and a

672 fixed amount of purified SV fraction (vesicle concentration; $26.7 \mathrm{nM}$, Protein

673 concentration; $99.7 \mathrm{ng} / \mu \mathrm{l}$ ) were subjected to quantitative western blot analysis (see also

674 Appendix Fig S5 for complete datasets and control experiments for Syb2). Signal

675 intensities of bands were quantified and plotted as a function of moles of GST-Stx7-N.

676 Using a standard curve, copy numbers of Stx7 / SV were estimated as $0.14 \pm 0.03$ (SD).

677 Data were obtained from experiments performed in quadruplicate, and the average and

678 standard deviation are shown.

679

680 Figure 3. Stx7-SEP-vesicles recycle preferentially upon high-frequency 681 stimulation.

682 a, Stimulus-frequency-dependent responses of Stx7-SEP in comparison to SypHy.

683 Neurons expressing respective SEP fusion constructs were subjected to sequential

684 stimulation ranging from 5 to $40 \mathrm{~Hz}$ (200 APs) at 5 min intervals. Left images are

685 representative images of SypHy (top), Stx7-SEP (bottom) at rest, at the end of

686 stimulation at $5 \mathrm{~Hz}, 10 \mathrm{~Hz}, 20 \mathrm{~Hz}$, and $40 \mathrm{~Hz}$, and upon application of $\mathrm{NH}_{4} \mathrm{Cl}$ at the end

687 of recordings. Right traces show representative traces of each SEP-fluorescence change

688 in boutons, indicated by arrows. Data were normalized to fluorescence signals upon

689 application of $\mathrm{NH}_{4} \mathrm{Cl}$ at the end of recordings. b, Experimental scheme to estimate

690 kinetics of exocytosis as well as sizes of total recycling SV pools. Neurons were

691 pretreated with $2 \mu \mathrm{M}$ bafilomycin A1 for $60 \mathrm{sec}$ and then stimulated with 600 APs at

692 different stimulation frequencies. After cessation of stimulation, $50 \mathrm{mM} \mathrm{NH} \mathrm{NCl}_{4}$ was

693 applied and fluorescence during $\mathrm{NH}_{4} \mathrm{Cl}$ application was used to normalized

694 fluorescence signals at individual boutons. c, Recycling pool of SypHy and Stx7-SEP. 
695 Cells expressing SypHy (left) and Stx7-SEP (right) were pretreated with $2 \mu \mathrm{M}$

696 bafilomycin A, and were stimulated with 600 APs at different frequencies (5, 10, 20 and

$69740 \mathrm{~Hz}$ ). d, Replots of SypHy (black) and Stx7-SEP (red) responses of the results in $\mathbf{c}$ as

698 a function of stimulus numbers (No. of AP).

699

700 Figure 4. Stx7-SEP specifies a recycling pool of SVs preferentially mobilized in the 701 presence of high $\mathrm{Ca}^{2+}$ concentration and actin polymerization.

702 a, Responses of Stx7-SEP (right) in the presence of normal (2 mM, black) and high (8 $703 \mathrm{mM}$, red) external $\mathrm{Ca}^{2+}$. For comparison, responses of SypHy under identical conditions 704 are shown in the left panel. b, Effects of latrunculin A (Lat-A, $5 \mu \mathrm{M}$; red) on responses 705 of SypHy (upper traces) and of Stx7-SEP (lower traces) elicited by $10-\mathrm{Hz}$ (left) and $70640-\mathrm{Hz}$ (right) stimulation. Control experiments without Lat-A in the respective 707 conditions are shown in black. c, Effects of Lat-A (red traces) on recycling of SypHy and Stx7-SEP in the presence of $8 \mathrm{mM}$ external $\mathrm{Ca}^{2+}$. Control experiments without LatA in the respective conditions are shown in black. d, Effects of Lat-A (red) on exocytosis of total recycling pool monitored by SypHy. Responses at $10 \mathrm{~Hz}, 600 \mathrm{APs}$

711 (left) and $20 \mathrm{~Hz}, 600 \mathrm{APs}$ (right) with bafilomycin treatment are shown. Control 712 experiments without Lat $-\mathrm{A}$ in the respective conditions are shown in black. e, 713 Quantitative comparisons of total recycling pool sizes and rise kinetics in (d). 714 Normalized fluorescence peaks (left graph) or the time constants of rise time $\left(\tau_{\text {exo }}\right.$ ) 715 (right graph) during the $10-\mathrm{Hz}$ or $20-\mathrm{Hz}$ stimulation are compared. All traces are 716 average traces from $>50-150$ boutons.

718 Figure 5. The N-terminal domain of Stx7 is required for proper sorting of Stx7 to 719 an SV subpopulation.

720 a, Schematic diagram of full length Stx7-SEP, Stx7-SEP lacking the N-terminal

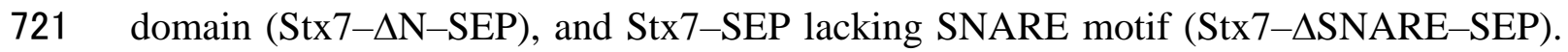

722 b, Distribution of Stx7-SEP, Stx7- $\mathrm{N}-\mathrm{SEP}$, and Stx7- $\Delta-$ SNARE-SEP in transfected 723 neurons. c,d The surface fraction of Stx7-SEP and truncated mutants (c) and vesicular $724 \mathrm{pHs}$ of vesicles carrying the respective SEPs (d). Note that vesicular pH of Stx7$725 \Delta$ SNARE-SEP could not be calculated, since it was exclusively expressed at the cell 726 surface (N.D. indicates 'not determined'). e, Responses of Stx7-SEP (black traces) and 727 Stx7- $\triangle \mathrm{N}-\mathrm{SEP}$ (red traces) upon 10-Hz (left panels) and $40-\mathrm{Hz}$ stimulation (right 
728 panels). f, Responses of Stx7- $\Delta \mathrm{N}-\mathrm{SEP}$ upon $10-\mathrm{Hz}$ stimulation in control (black) and

729 after TeNT treatment (red). g, Responses of Stx7- $\mathrm{N}-\mathrm{SEP}$ upon $40-\mathrm{Hz}$ stimulation in

730 control (black) and after Lat-A treatment (red).

731

732 Figure 6. Overexpression of Stx7-NTD slowed SypHy responses only during HFS.

733 a, Schematic diagram of SypHy and SypHy co-expressed with Stx7-NTD. Stx7-NTD

734 was placed after a P2A sequence so that all SypHy-positive cells always co-expressed

735 Stx7-NTD. b, Effect of Stx7-NTD on the total recycling pool monitored by SypHy

736 responses at $10 \mathrm{~Hz}, 600 \mathrm{APs}$ (left) and $20 \mathrm{~Hz}, 600 \mathrm{APs}$ (right) after bafilomycin

737 treatment. c, Quantification of total recycling pool sizes (left) and time constants of rise

738 time (right, $\tau_{\text {exo) }}$ in $(\mathbf{b})$. d, Pretreatment with Lat-A did not further reduce release

739 kinetics on Stx7-NTD overexpression. Responses of SypHy with Stx7-NTD in the

740 presence of Lat-A (blue) was compared with control (SypHy only, black) and SypHy

741 with Stx7-NTD (red) in response to at $20 \mathrm{~Hz}$, 600APs. A right graph shows time

742 constants of the rise time $\left(\tau_{\text {exo }}\right)$. SypHy responses with Stx7-NTD (red) and Stx7-NTD

$743+$ Lat-A (blue) did not differ significantly $(p>0.05)$. 


\section{Acknowledgements}

748 We thank all members of the Takamori laboratory and Dr. Tetsuya Hori for helpful

749 discussions. We thank Steven D. Aird for editing the manuscript. This work was

750 supported by grants from JSPS KAKENHI (16H04675, 19H03330), the JSPS

751 Core-to-Core Program, A. Advanced Research Networks grant, and a Grant from Naito

752 Foundation to ST, and a grant from JSPS KAKENHI (18K06473) to YM.

753

754

\section{Author contributions}

755 YM and ST conceived the study. YM, KT and YF performed experiments and analyzed

756 the data. ST wrote the manuscript with assistance from YM.

757

758

759

760 
761

762

763

764

765

766

767

768

769

770

771

772

773

774

775

776

777

778

779

780

781

782

783

784

785

786

\section{References}

Alabi AA \& Tsien RW (2012) Synaptic vesicle pools and dynamics. Cold Spring Harb Perspect Biol 4: a013680

Antonin W, Dulubova I, Arac D, Pabst S, Plitzner J, Rizo J \& Jahn R (2002) The $\mathrm{N}$-terminal domains of syntaxin 7 and vtilb form three-helix bundles that differ in their ability to regulate SNARE complex assembly. J. Biol. Chem. 277: 36449-36456

Antonin W, Holroyd C, Fasshauer D, Pabst S, Von Mollard GF \& Jahn R (2000) A SNARE complex mediating fusion of late endosomes defines conserved properties of SNARE structure and function. EMBO J. 19: 6453-6464

Chanaday NL \& Kavalali ET (2018) Optical detection of three modes of endocytosis at hippocampal synapses. Elife 7:

Chen C \& Okayama H (1987) High-efficiency transformation of mammalian cells by plasmid DNA. Mol. Cell. Biol. 7: 2745-2752

Egashira Y, Takase M \& Takamori S (2015) Monitoring of vacuolar-type H+ ATPase-mediated proton influx into synaptic vesicles. J. Neurosci. 35: 3701-3710

Egashira Y, Takase M, Watanabe S, Ishida J, Fukamizu A, Kaneko R, Yanagawa Y \& Takamori S (2016) Unique $\mathrm{pH}$ dynamics in GABAergic synaptic vesicles illuminates the mechanism and kinetics of GABA loading. Proc. Natl. Acad. Sci. U.S.A. 113: 10702-10707

Emperador-Melero J, Huson V, van Weering J, Bollmann C, Fischer von Mollard G, Toonen RF \& Verhage M (2018) Vtila/b regulate synaptic vesicle and dense core vesicle secretion via protein sorting at the Golgi. Nat Commun 9: 3421

Granseth B, Odermatt B, Royle SJ \& Lagnado L (2006) Clathrin-mediated endocytosis is the dominant mechanism of vesicle retrieval at hippocampal synapses. Neuron 51: 773-786 
787 He J, Johnson JL, Monfregola J, Ramadass M, Pestonjamasp K, Napolitano G, Zhang J

788 \& Catz SD (2016) Munc13-4 interacts with syntaxin 7 and regulates late endosomal 789 maturation, endosomal signaling, and TLR9-initiated cellular responses. Mol. Biol. Cell $790 \quad$ 27: $572-587$

791 He L, Xue L, Xu J, McNeil BD, Bai L, Melicoff E, Adachi R \& Wu L-G (2009)

792 Calcium/synaptotagmin-evoked compound fusion increases quantal size and synaptic

793 strength. Nature 459: 93-97

794 Holroyd C, Kistner U, Annaert W \& Jahn R (1999) Fusion of Endosomes Involved in 795 Synaptic Vesicle Recycling. Mol Biol Cell 10: 3035-3044

796 Hoopmann P, Punge A, Barysch SV, Westphal V, Bückers J, Opazo F, Bethani I, 797 Lauterbach MA, Hell SW \& Rizzoli SO (2010) Endosomal sorting of readily releasable 798 synaptic vesicles. Proc. Natl. Acad. Sci. U.S.A. 107: 19055-19060

799 Hua Z, Leal-Ortiz S, Foss SM, Waites CL, Garner CC, Voglmaier SM \& Edwards RH 800 (2011) v-SNARE composition distinguishes synaptic vesicle pools. Neuron 71: 474 801487

802 Ibata K, Kono M, Narumi S, Motohashi J, Kakegawa W, Kohda K \& Yuzaki M (2019)

803 Activity-Dependent Secretion of Synaptic Organizer Cbln1 from Lysosomes in Granule 804 Cell Axons. Neuron 102: 1184-1198.e10

805 Ikeda K \& Bekkers JM (2009) Counting the number of releasable synaptic vesicles in a 806 presynaptic terminal. Proc. Natl. Acad. Sci. U.S.A. 106: 2945-2950

807 Jahn R \& Scheller RH (2006) SNAREs--engines for membrane fusion. Nat. Rev. Mol. 808 Cell Biol. 7: 631-643

809 Jurado S, Goswami D, Zhang Y, Molina AJM, Südhof TC \& Malenka RC (2013) LTP 810 requires a unique postsynaptic SNARE fusion machinery. Neuron 77: 542-558

811 Kim SH \& Ryan TA (2009) Synaptic vesicle recycling at CNS snapses without AP-2. J.

812 Neurosci. 29: 3865-3874 
813 Kim SH \& Ryan TA (2010) CDK5 serves as a major control point in neurotransmitter

814 release. Neuron 67: 797-809

815 Kononenko NL, Puchkov D, Classen GA, Walter AM, Pechstein A, Sawade L, Kaempf

816 N, Trimbuch T, Lorenz D, Rosenmund C, Maritzen T \& Haucke V (2014)

817 Clathrin/AP-2 mediate synaptic vesicle reformation from endosome-like vacuoles but

818 are not essential for membrane retrieval at central synapses. Neuron 82: 981-988

819 Lee JS, Ho W-K \& Lee S-H (2012) Actin-dependent rapid recruitment of reluctant 820 synaptic vesicles into a fast-releasing vesicle pool. Proc. Natl. Acad. Sci. U.S.A. 109: $821 \quad$ E765-774

822 Liu H, Bai H, Hui E, Yang L, Evans CS, Wang Z, Kwon SE \& Chapman ER (2014)

823 Synaptotagmin 7 functions as a Ca2+-sensor for synaptic vesicle replenishment. eLife 3:

$824 \quad \mathrm{e} 01524$

825 Matsui T, Jiang P, Nakano S, Sakamaki Y, Yamamoto H \& Mizushima N (2018)

826 Autophagosomal YKT6 is required for fusion with lysosomes independently of syntaxin

827 17. J. Cell Biol. 217: 2633-2645

828 Miesenböck G, De Angelis DA \& Rothman JE (1998) Visualizing secretion and 829 synaptic transmission with $\mathrm{pH}$-sensitive green fluorescent proteins. Nature 394: 192$830 \quad 195$

831 Mitchell SJ \& Ryan TA (2004) Syntaxin-1A is excluded from recycling synaptic vesicles at nerve terminals. J. Neurosci. 24: 4884-4888

833 Mullock BM, Smith CW, Ihrke G, Bright NA, Lindsay M, Parkinson EJ, Brooks DA, 834 Parton RG, James DE, Luzio JP \& Piper RC (2000) Syntaxin 7 is localized to late 835 endosome compartments, associates with Vamp 8, and Is required for late 836 endosome-lysosome fusion. Mol. Biol. Cell 11: 3137-3153

837 Nicholson-Fish JC, Kokotos AC, Gillingwater TH, Smillie KJ \& Cousin MA (2015) 838 VAMP4 Is an Essential Cargo Molecule for Activity-Dependent Bulk Endocytosis. $839 \quad$ Neuron 88: 973-984 
840 Onoa B, Li H, Gagnon-Bartsch JA, Elias LAB \& Edwards RH (2010) Vesicular

841 monoamine and glutamate transporters select distinct synaptic vesicle recycling

842 pathways. J. Neurosci. 30: 7917-7927

843 Piriya Ananda Babu L, Wang H-Y, Eguchi K, Guillaud L \& Takahashi T (2020)

844 Microtubule and Actin Differentially Regulate Synaptic Vesicle Cycling to Maintain

845 High-Frequency Neurotransmission. J. Neurosci. 40: 131-142

846 Raingo J, Khvotchev M, Liu P, Darios F, Li YC, Ramirez DMO, Adachi M, Lemieux P,

847 Toth K, Davletov B \& Kavalali ET (2012) VAMP4 directs synaptic vesicles to a pool

848 that selectively maintains asynchronous neurotransmission. Nat. Neurosci. 15: 738-745

849 Ramirez DMO, Khvotchev M, Trauterman B \& Kavalali ET (2012) Vtila identifies a 850 vesicle pool that preferentially recycles at rest and maintains spontaneous 851 neurotransmission. Neuron 73: 121-134

852 Rizzoli SO, Bethani I, Zwilling D, Wenzel D, Siddiqui TJ, Brandhorst D \& Jahn R 853 (2006) Evidence for early endosome-like fusion of recently endocytosed synaptic $854 \quad$ vesicles. Traffic 7: 1163-1176

855 Rizzoli SO \& Betz WJ (2005) Synaptic vesicle pools. Nat. Rev. Neurosci. 6: 57-69

856 Sakaba T \& Neher E (2001) Calmodulin mediates rapid recruitment of fast-releasing 857 synaptic vesicles at a calyx-type synapse. Neuron 32: 1119-1131

858 Sakaba T \& Neher E (2003) Involvement of actin polymerization in vesicle recruitment 859 at the calyx of Held synapse. J. Neurosci. 23: 837-846

860 Sankaranarayanan S, Atluri PP \& Ryan TA (2003) Actin has a molecular scaffolding, 861 not propulsive, role in presynaptic function. Nat. Neurosci. 6: 127-135

862 Sankaranarayanan S, De Angelis D, Rothman JE \& Ryan TA (2000) The use of 863 pHluorins for optical measurements of presynaptic activity. Biophys. J. 79: 2199-2208

864 Schiavo G, Benfenati F, Poulain B, Rossetto O, Polverino de Laureto P, DasGupta BR 865 \& Montecucco C (1992) Tetanus and botulinum-B neurotoxins block neurotransmitter 866 release by proteolytic cleavage of synaptobrevin. Nature 359: 832-835 
867 Schoch S, Deák F, Königstorfer A, Mozhayeva M, Sara Y, Südhof TC \& Kavalali ET

868 (2001) SNARE function analyzed in synaptobrevin/VAMP knockout mice. Science

869 294: $1117-1122$

870 Shimojo M, Courchet J, Pieraut S, Torabi-Rander N, Sando R, Polleux F \& Maximov A

871 (2015) SNAREs Controlling Vesicular Release of BDNF and Development of Callosal

872 Axons. Cell Rep 11: 1054-1066

873 Silm K, Yang J, Marcott PF, Asensio CS, Eriksen J, Guthrie DA, Newman AH, Ford

874 CP \& Edwards RH (2019) Synaptic Vesicle Recycling Pathway Determines

875 Neurotransmitter Content and Release Properties. Neuron 102: 786-800.e5

876 Sudhof TC (2004) The synaptic vesicle cycle. Annu. Rev. Neurosci. 27: 509-547

877 Takamori S, Holt M, Stenius K, Lemke EA, Grønborg M, Riedel D, Urlaub H, Schenck

878 S, Brügger B, Ringler P, Müller SA, Rammner B, Gräter F, Hub JS, De Groot BL,

879 Mieskes G, Moriyama Y, Klingauf J, Grubmüller H, Heuser J, et al (2006) Molecular 880 anatomy of a trafficking organelle. Cell 127: 831-846

881 Voglmaier SM, Kam K, Yang H, Fortin DL, Hua Z, Nicoll RA \& Edwards RH (2006)

882 Distinct endocytic pathways control the rate and extent of synaptic vesicle protein 883 recycling. Neuron 51: 71-84

884 Ward DM, Pevsner J, Scullion MA, Vaughn M \& Kaplan J (2000) Syntaxin 7 and 885 VAMP-7 are soluble N-ethylmaleimide-sensitive factor attachment protein receptors 886 required for late endosome-lysosome and homotypic lysosome fusion in alveolar 887 macrophages. Mol. Biol. Cell 11: 2327-2333

888 Wilhelm BG, Mandad S, Truckenbrodt S, Kröhnert K, Schäfer C, Rammner B, Koo SJ, 889 Claßen GA, Krauss M, Haucke V, Urlaub H \& Rizzoli SO (2014) Composition of 890 isolated synaptic boutons reveals the amounts of vesicle trafficking proteins. Science 891 344: 1023-1028

892 Wu X-S, Lee SH, Sheng J, Zhang Z, Zhao W-D, Wang D, Jin Y, Charnay P, Ervasti JM 893 \& Wu L-G (2016) Actin Is Crucial for All Kinetically Distinguishable Forms of 894 Endocytosis at Synapses. Neuron 92: 1020-1035 
bioRxiv preprint doi: https://doi.org/10.1101/2020.08 13.249672; this version posted August 14, 2020. The copyright holder for this preprint (which was not certified by peer review) is the author/funder, who has granted bioRxiv a license to display the preprint in perpetuity. It is made available under aCC-BY 4.0 International license. 
A
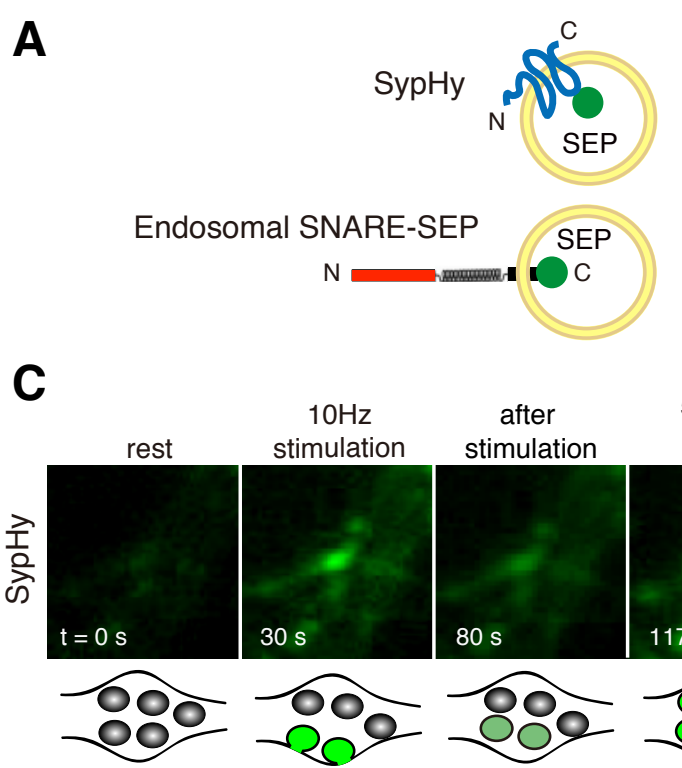

$\mathbf{E}$

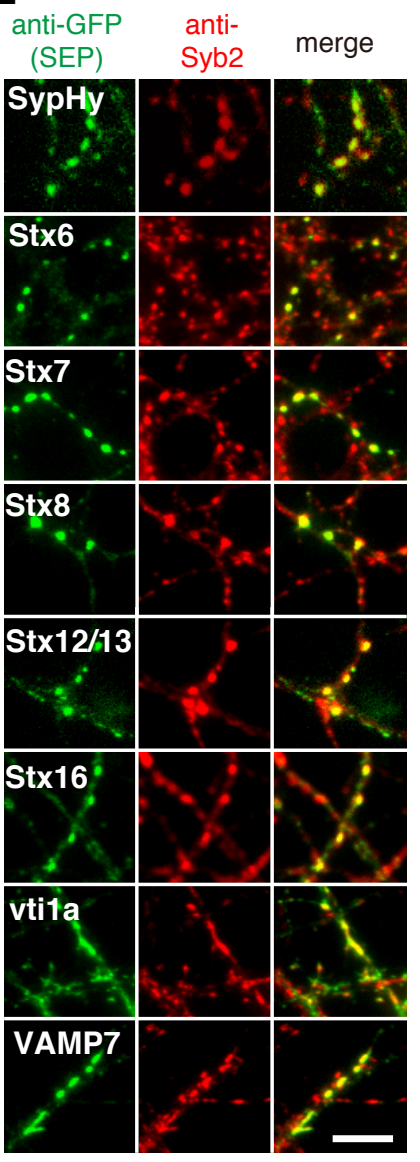

B
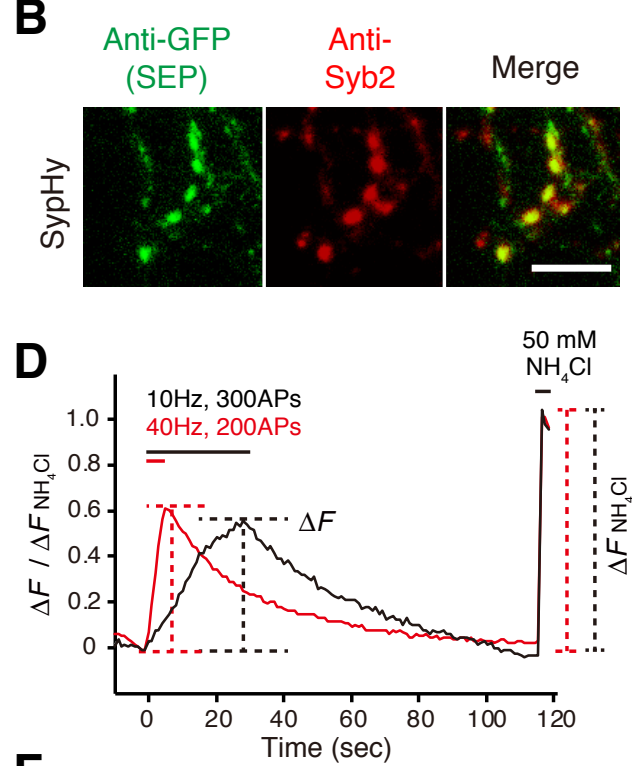

$\mathbf{F}$

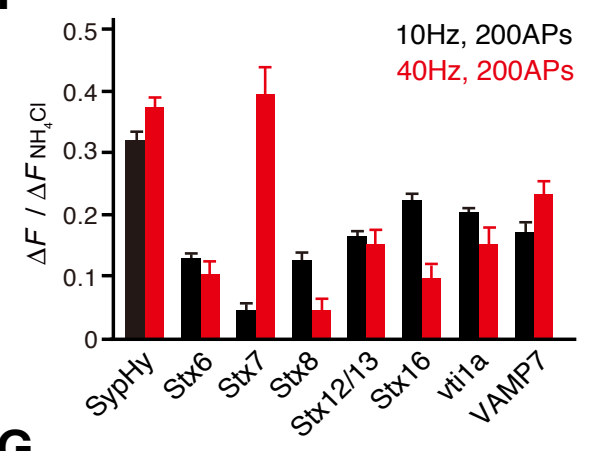

G
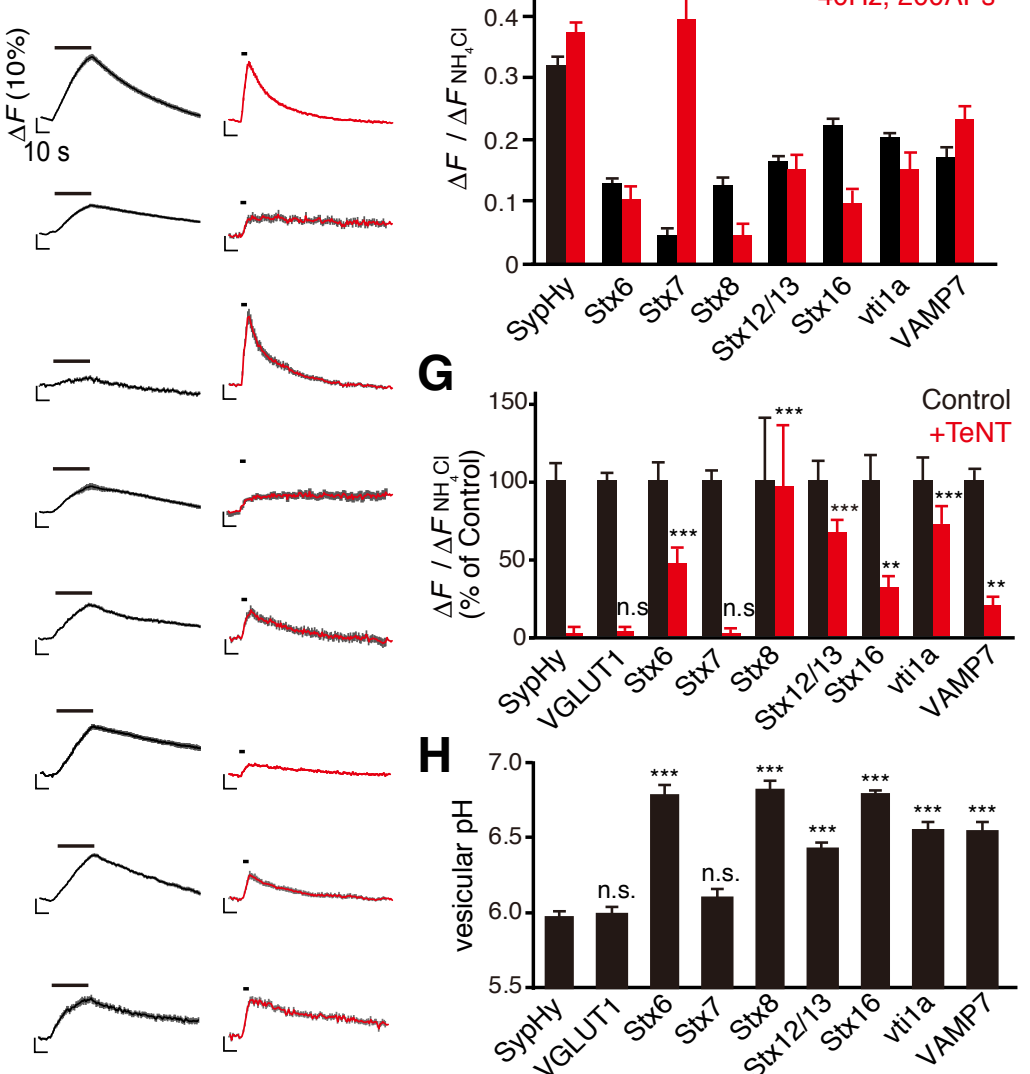

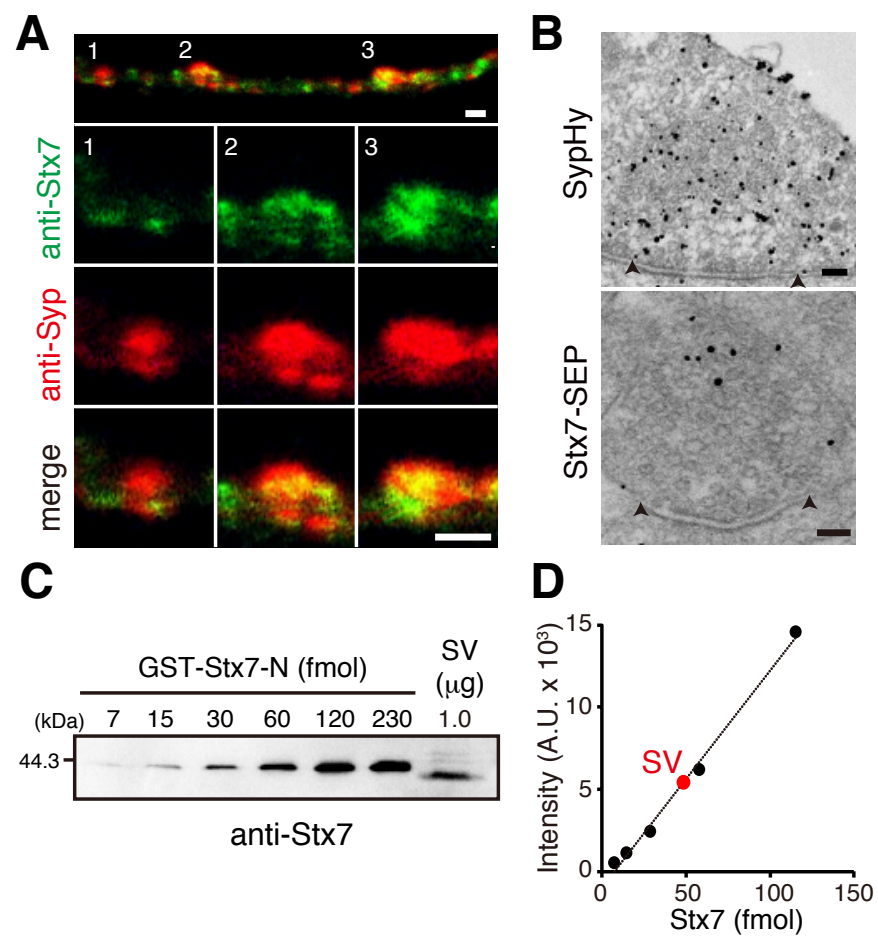
A
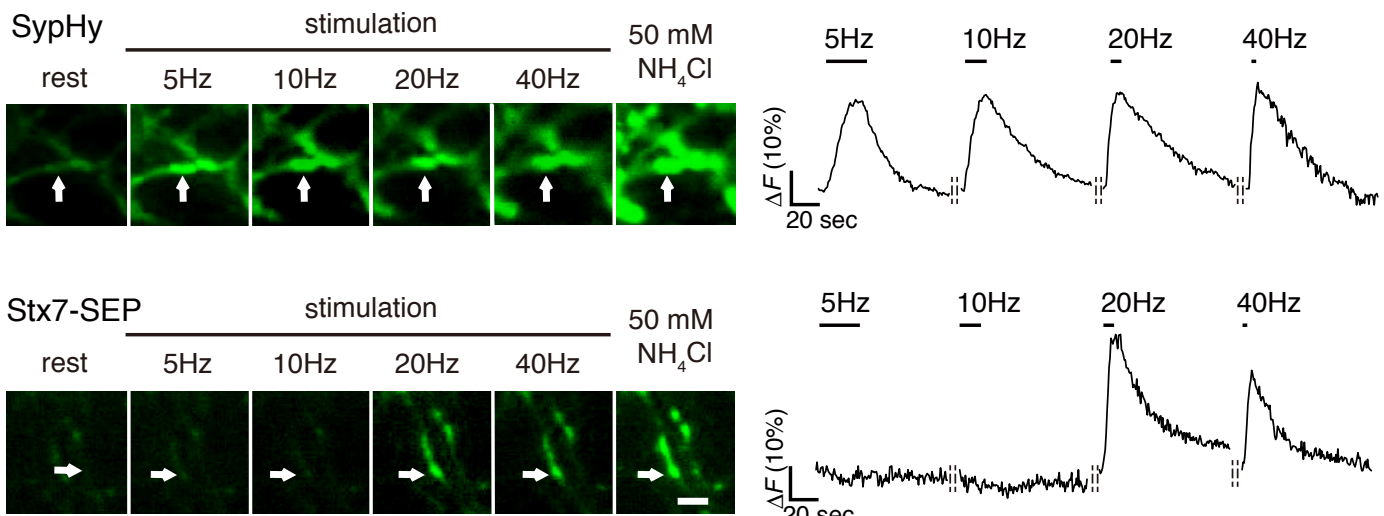

mulation

$50 \mathrm{mM}$

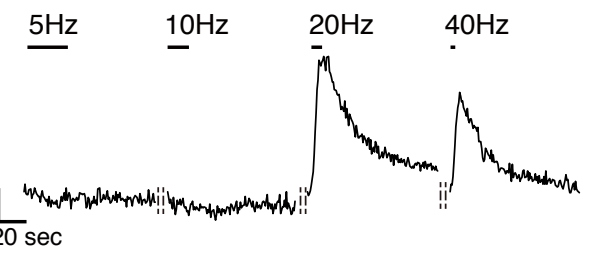

B SypHy

C

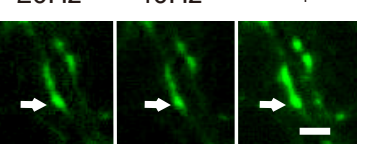

$20 \mathrm{sec}$

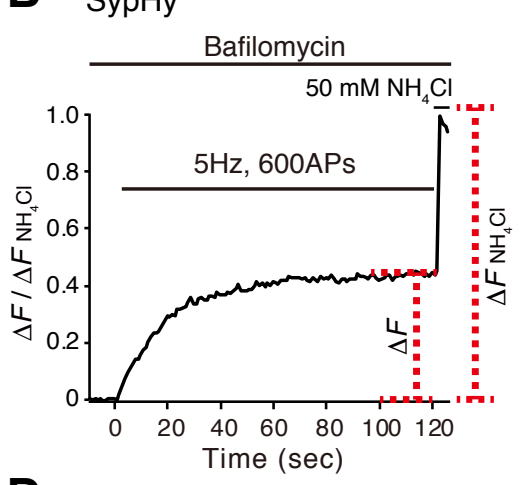

SypHy

Stx7-SEP
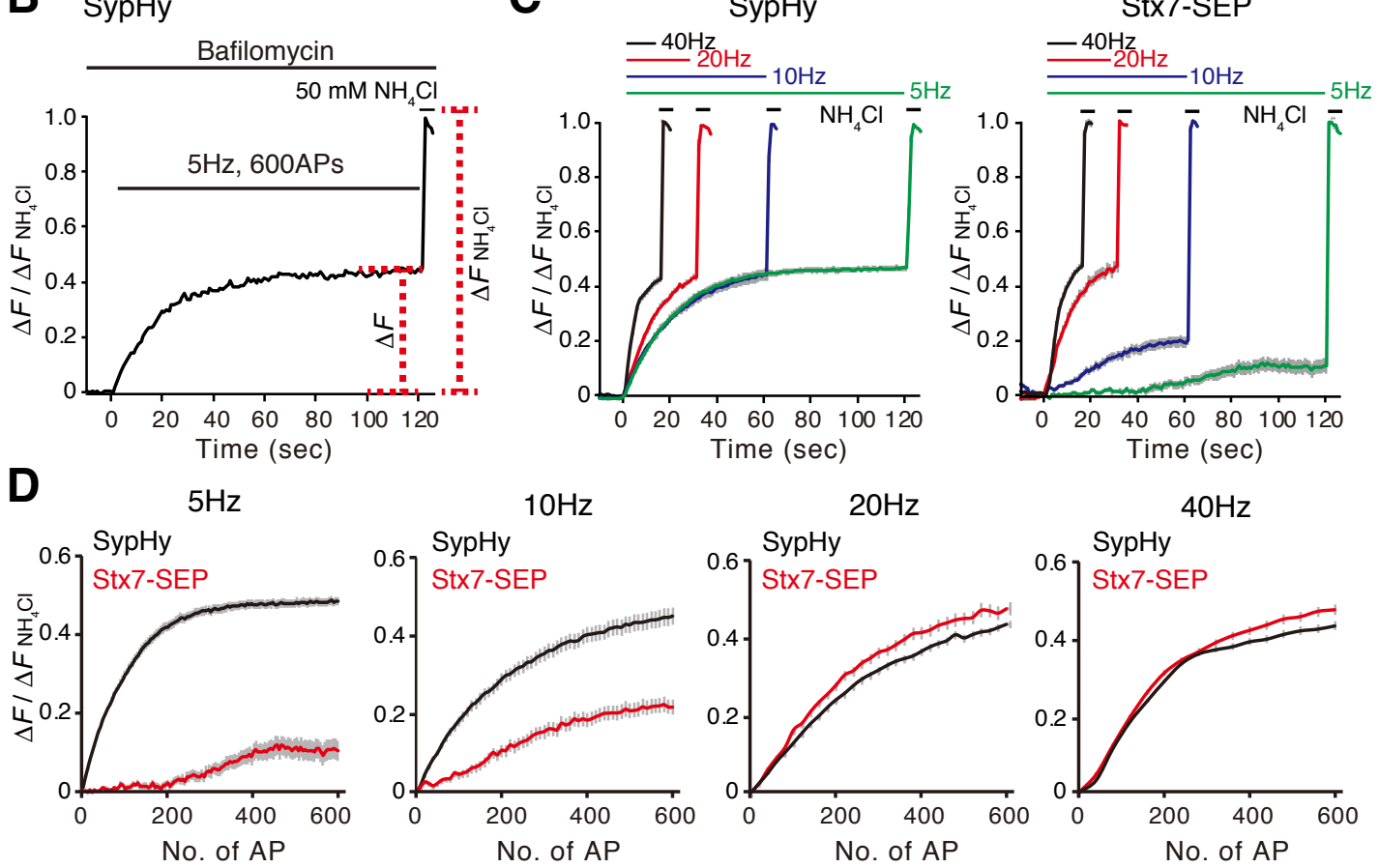
A

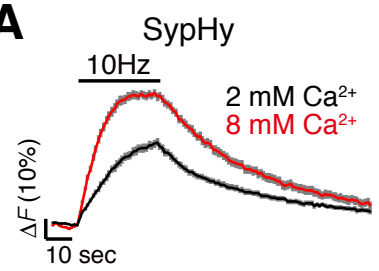

B
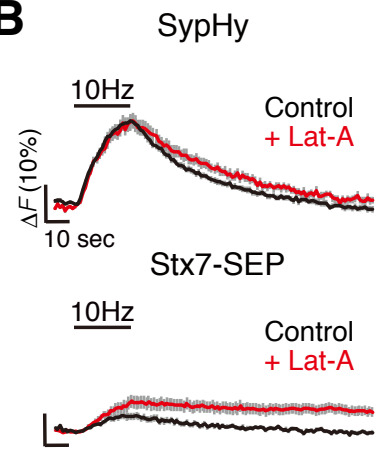

C SypHy $\left(8 \mathrm{mM} \mathrm{Ca}^{2+}\right)$
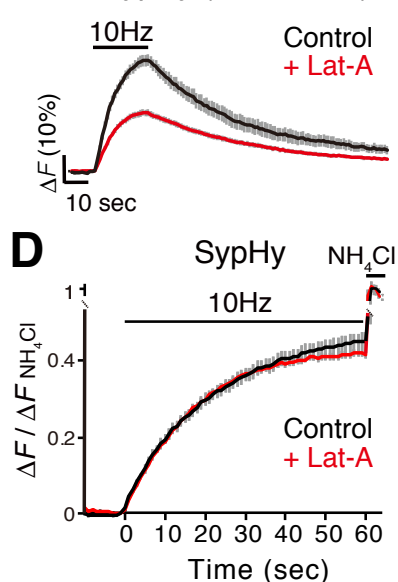

$\mathbf{E}$

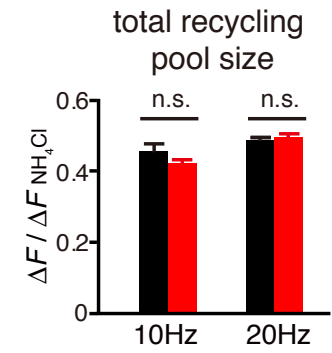

Stx7-SEP

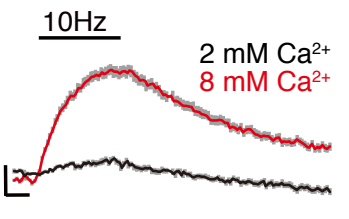

SypHy

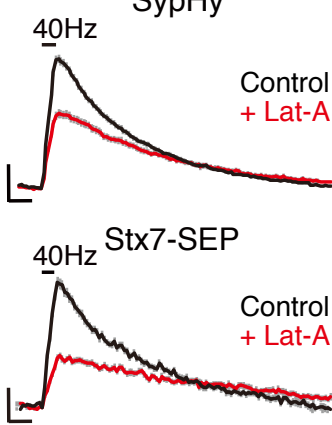

Stx7-SEP (8 mM Ca ${ }^{2+}$ )
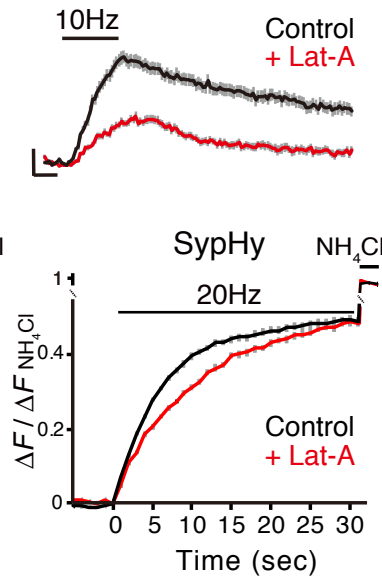

rise kinetics

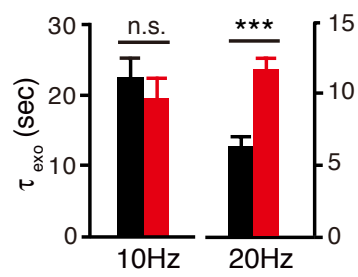


A
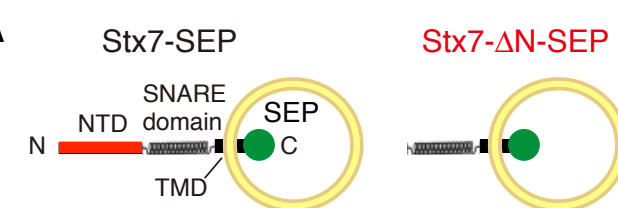

B

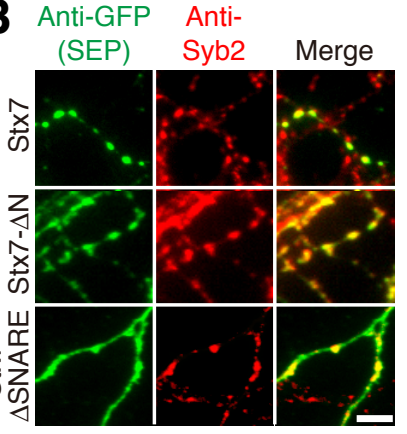

E
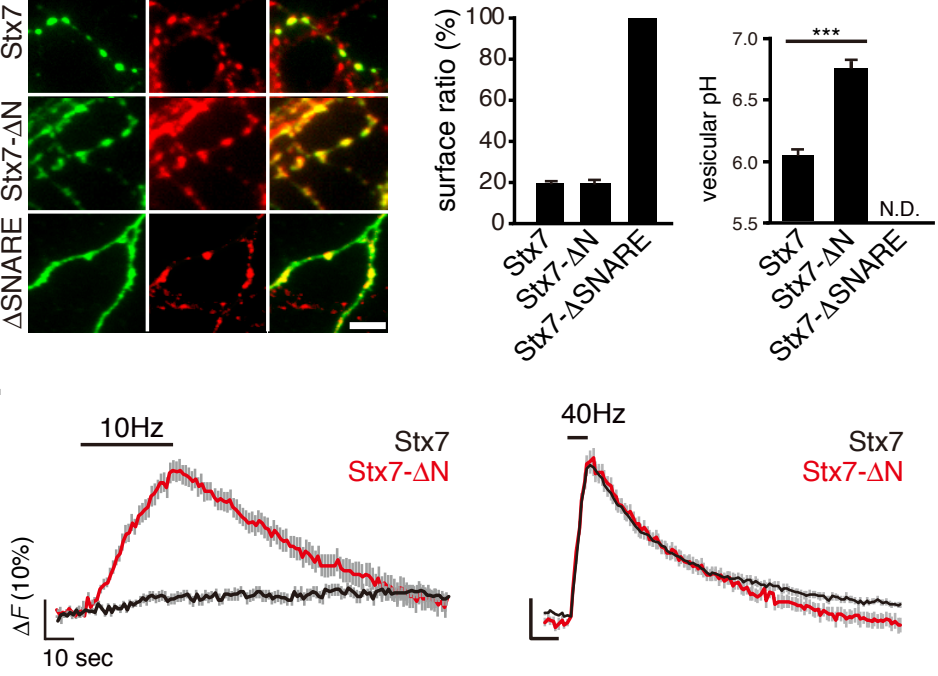

$\mathbf{F}$

Stx7- $\Delta N-S E P$

G
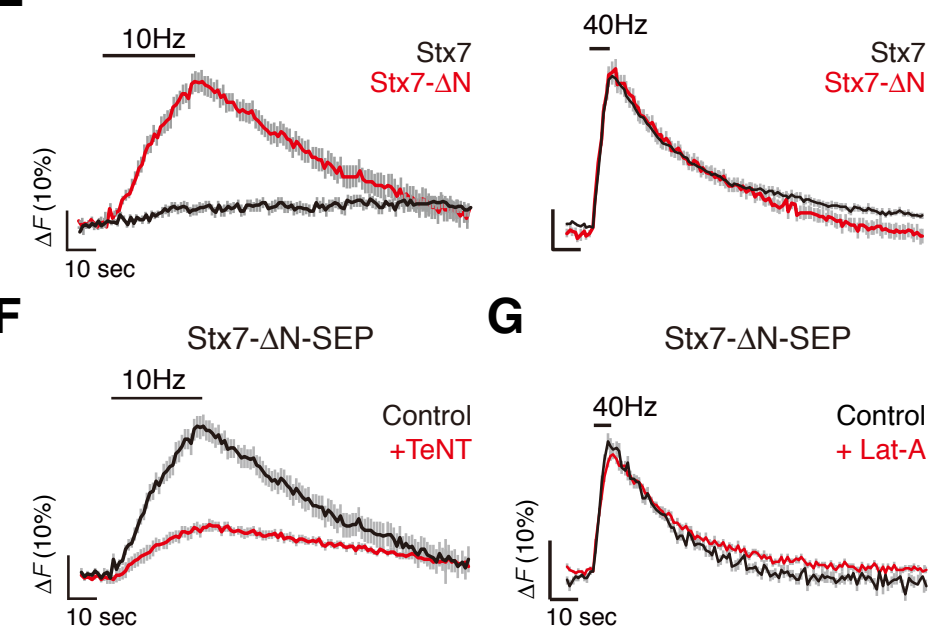

G Stx7- $\triangle N-S E P$

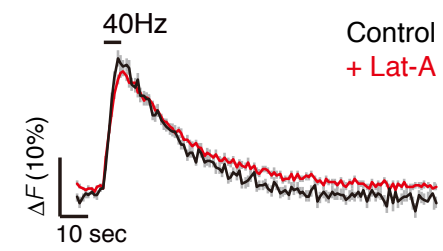

Mori et al Fig. 5 
A

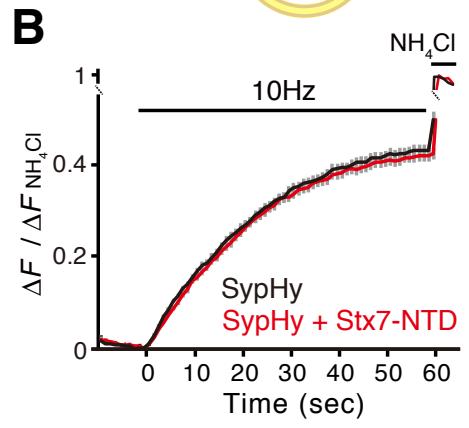

C
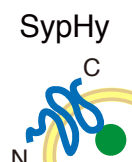

total recycling pool size

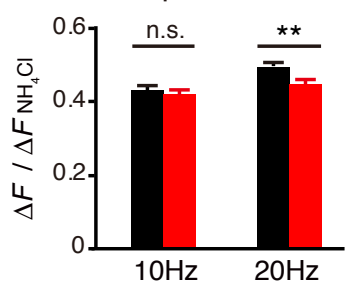

SypHy + Stx7-NTD
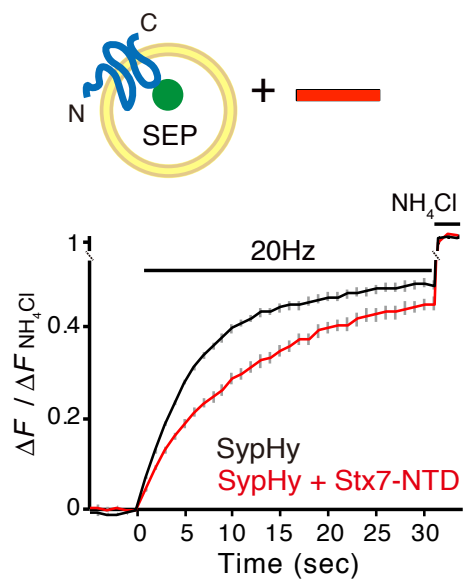

rise kinetics

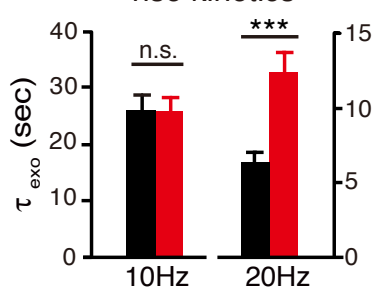

rise kinetics
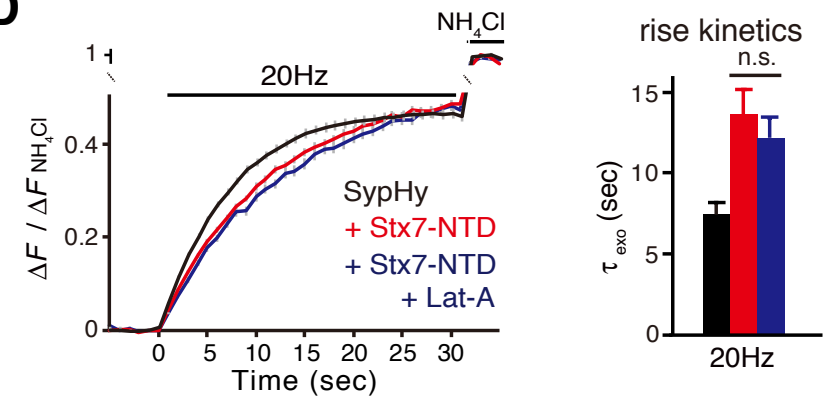

$\mathbf{E}$

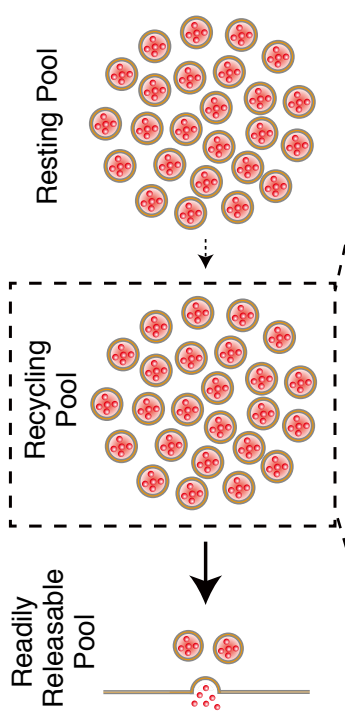

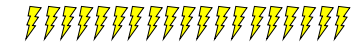

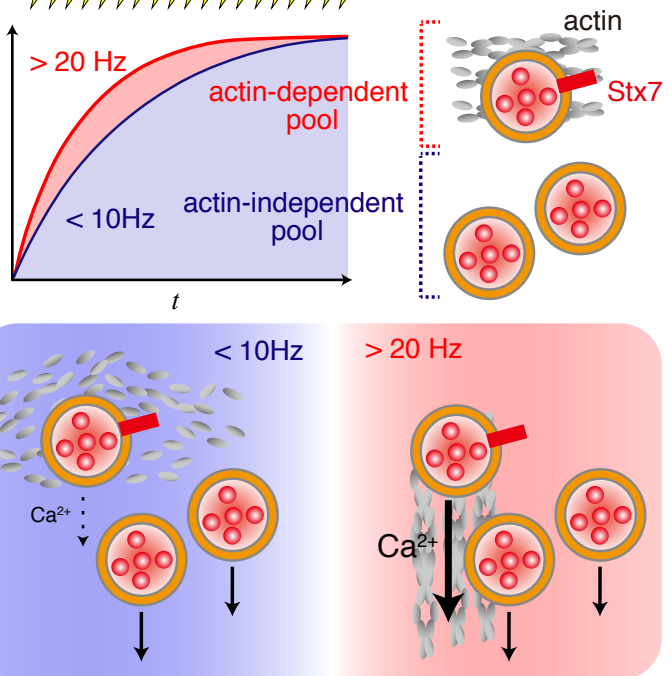

